Research Article

\title{
Controlling the PVTOL Aircraft System with an Inverted Pendular Load by means of Nested Saturation Functions and GPI Controller
}

\author{
Cesar Alejandro Villaseñor Rios $\mathbb{D}$, Octavio Gutiérrez-Frías $\mathbb{D}$, Carlos Aguilar-Ibanez $\mathbb{D}$, \\ and Miguel S. Suarez-Castanon $(\mathbb{D}$
}

Instituto Politécnico Nacional, Mexico City, Mexico

Correspondence should be addressed to Octavio Gutiérrez-Frías; ogutierrezf@ipn.mx

Received 24 September 2021; Accepted 30 October 2021; Published 19 November 2021

Academic Editor: Baltazar Aguirre Hernandez

Copyright (c) 2021 Cesar Alejandro Villaseñor Rios et al. This is an open access article distributed under the Creative Commons Attribution License, which permits unrestricted use, distribution, and reproduction in any medium, provided the original work is properly cited.

\begin{abstract}
This paper presents a control scheme that allows height position regulation and stabilization for an unmanned planar vertical takeoff and landing aircraft system with an inverted pendular load. The proposed controller consists of nested saturations and a generalized proportional integral (GPI). The GPI controls the aircraft height and the roll attitude; the latter is used as the fictitious input control. Next, the system is reduced through linear transformations, expressing it as an integrator chain with a nonlinear perturbation. Finally, the nested saturation function-based controller stabilizes the aircraft's horizontal position and the pendulum's angle. Obtaining the control approach was a challenging task due to the underactuated nature of the aircraft, particularly ensuring the pendulum's upright position. The stability analysis was based on the second method of Lyapunov using a simple candidate function. The numerical simulation confirmed the control strategy's effectiveness and performance. Additionally, the numerical simulation included a comparison against a PD controller, where its corresponding performance indexes were estimated, revealing that our controller had a better response in the presence of unknown disturbances.
\end{abstract}

\section{Introduction}

The control of underactuated mechanical systems is a widely studied field and continuously increases knowledge, mainly because controlling this kind of system is challenging since it has fewer controllers than degrees of freedom. The inverted pendulum system is a classic example of an underactuated system. It consists of a freely spinning load around an axis and attached to a base that freely moves forward and backward-multiple authors have proposed control laws to stabilize this kind of system in its inverted position. Block and Spong [1] proposed the partial feedback collocated and noncollocated control law for the stabilization around the origin of the acrobot and the pendubot. Fantoni and Lozano [2] proposed a nested saturation control for the wheeled inverted pendulum that enables stabilization around the origin. Ibañez and Frias [3] proposed a nested saturation control for the nonlinear perturbed wheeled inverted pendulum, expressed it as a chain of integrators. The proposed control demonstrates asymptotical stability around the origin through the Lyapunov method when the pendulum angle is in the upper half-plane. The Furuta pendulum is another challenging system that has attracted the attention of several researchers, who have presented many interesting approaches to control this kind of pendulum. For instance, in [4], the authors proposed a Lyapunov-based control method for the stabilization around the origin, Furuta pendulum stabilization around the origin, while in [5], the authors introduced an active disturbance rejectionbased control and its stability analysis. Another exciting example of underactuated systems is the unmanned aerial vehicle (UAV) such that this kind of system has been of high interest in the present century because of wide applications for different fields such as farming, photography, exploration, and military [6-8]. A typical example of a UAV is the planar vertical takeoff 
and landing (PVTOL) aircraft, a simplified model of the actual vertical takeoff and landing aircraft [9], which encompasses almost all the dynamics found in real UAVs. The PVTOL has been used as a suitable benchmark to test new and existing controllers because it behaves like the well-known quadrotor in a two-dimensional plane. There exist many works that tackle the PVTOL stabilization problem. For instance, Aguilar-Ibanez et al. [10] proposed a control scheme using controlled Lagrangian for PVTOL stabilization. Fantoni et al. [11] introduced a control scheme using the PVTOL attitude as fictitious control and nested saturation technique for the stabilization at the origin. In [12], Lozano et al. showed that once controlling the PVTOL aircraft height, the system resembles an inverted pendulum, and using a change of coordinates, it can be seen as an integrator chain with nonlinear disturbance. Also, in this study, the authors present a nested saturation-based control and demonstrated asymptotic stabilization at the origin. In [13], based on the image-based visual servoing method and the backstepping technique, the authors presented a novel control strategy to force a vertical takeoff and landing (VTOL) aircraft. Works $[14,15]$ can be valuable sources for the readers interested in this problem.

Combining the inverted pendulum and UAV systems adds a degree of freedom, obtaining a system noneasy to stabilize. Hehn et al. [16] proposed this problem in 2011 and named it the flying inverted pendulum, consisting of an inverted pendulum attached to a quadcopter. The control goals of this study are stabilization at the origin and tracking a circular trajectory for the quadcopter, using linear quadratic regulator (LQR) control in both cases. The fact that the pendulum weights less than $5 \%$ of the UAV weight allows us to separate its corresponding dynamics. Some works found in the specialized literature deal with the control of the flying inverted pendulum using different control ideas [17-20], and some others consider the control of UAVs carrying loads (commonly known as the flying crane). This problem is closely related and relevant to the central control problem in this study. For instance, Nicotra et al. [21] showed that the linearized model of a quadcopter with a suspended load could be stabilized at the origin using nested saturation functions. Pizetta et al. in [22] proposed a total feedback control with an auxiliary controller to accomplish tracking trajectory; almost all the references therein were developed to test the PVTOL system indoors, mainly to avoid counteracting the undesirable effect of the wind, which is not an easy task. However, techniques for nonlinear systems can be applied to obtain robust controllers, as the controller developed in this study. Of these techniques, perhaps the most used are backstepping control $[23,24]$, fuzzy control [25-27], active disturbance rejection control approach [28], and others [29]. To the authors' knowledge, a general solution for stabilizing this type of system has not yet been reported in the literature.

In this context, we propose a control scheme for a PVTOL aircraft system with an inverted pendular load (PVTOL-ASIPL). This scheme mainly consists of a generalized proportional integral (GPI) controller and a nested saturation-based control. The GPI controller accomplishes height and roll attitude control, using the roll attitude angle as fictitious input control. Then, proposing a set of convenient linear transformations and reducing the system can be expressed as a chain of integrators with a nonlinear perturbation. Finally, we design a nested saturation function-based controller to stabilize the horizontal position and the pendulum angle. Applying the second method of Lyapunov assures boundedness of the whole state and asymptotic convergence to the origin.

The main contributions of this study are as follows:

(i) An algorithm control that uses a fictitious control, and we propose a combination of a GPI controller and a controller-based saturation for the takeoff and landing maneuvers

(ii) A set of convenient transformations, in which a high-order system can be expressed as a various low-order system

(iii) A control strategy for the PVTOL aircraft system with an inverted pendular load controls the height, roll attitude, horizontal position, and roll angle simultaneously, even in the presence of exogenous disturbance

The organization of the rest of this study is as follows. Section 2 introduces the model of PVTOL-ASIPL externally perturbed, obtained from the Euler-Lagrange formalism. Section 3 develops the control scheme design, while Section 4 presents the results of the numerical simulations. Finally, Section 5 is devoted to the concluding remarks and future work.

\section{Dynamic Model}

This section presents the dynamic model of the PVTOL aircraft system with an inverted pendular load (see Figure 1). The dynamic equations were obtained by the Euler-Lagrange formalism as follows:

$$
\mathscr{L}=E_{k}-E_{p},
$$

where $E_{k}$ and $E_{p}$ are, respectively, the system kinetic and potential energies. Besides, the inverted pendular load base is in the PVTOL aircraft center of mass, $P_{v}=\left(x_{v}, y_{v}\right), \alpha$ is defined as the angle between the PVTOL aircraft and the horizontal axis, and $\theta$ is the angle between the inverted pendular load and the vertical axis. Finally, the inverted pendular load center of mass $\left(P_{P}=\left(x_{p}, y_{p}\right)\right)$ is defined as

$$
\begin{aligned}
& x_{p}=x_{v}-l_{p} \sin \theta, \\
& y_{p}=y_{v}+l_{p} \cos \theta .
\end{aligned}
$$

Therefore, Lagrangian of the system can be expressed as

$$
\mathscr{L}=\frac{m_{v} \dot{P}_{v} \dot{P}_{v}^{\top}}{2}+\frac{m_{p} \dot{P}_{p} \dot{P}_{p}^{\top}}{2}+\frac{\alpha^{2} i_{v}}{2}+\frac{\theta^{2} i_{p}}{2}-m_{v} g y_{v}-m_{p} g y_{p},
$$

where $m_{v}$ is the PVTOL aircraft mass, $m_{p}$ is the pendular load mass, $g$ is the gravity force, $i_{v}$ is the PVTOL aircraft inertia, and $i_{p}$ is the inverted pendular load inertia.

The Euler-Lagrange equations of motion for the PVTOL-ASIPL system are in the form of 


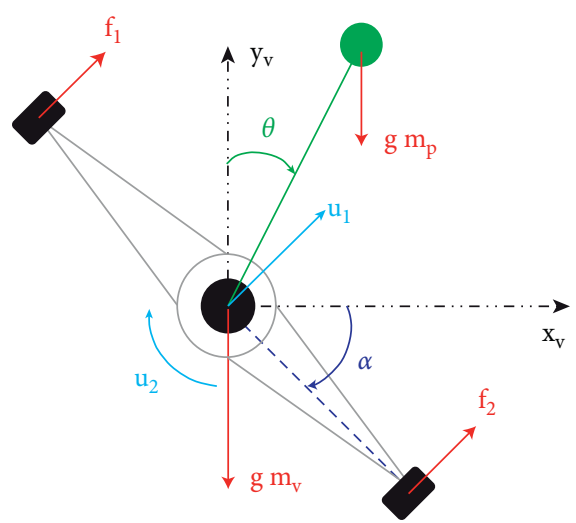

Figure 1: The PVTOL aircraft system with an inverted pendular load.

$$
\frac{d}{\mathrm{~d} t} \frac{\partial \mathscr{L}}{\partial \dot{q}}-\frac{\partial \mathscr{L}}{\partial q}=\tau+D
$$

where $q=\left(x_{v}, y_{v}, \alpha, \theta\right)$ is the generalized coordinate vector, $\tau$ is the input control vector, and $D$ is the external disturbance vector; without loss of generality, $i_{v}=1$ and $i_{p}=0$.

Developing Euler-Lagrange equation (4) leads to

$$
\begin{aligned}
l_{p} m_{p} \sin (\theta) \dot{\theta}^{2}+\left(m_{v}+m_{p}\right) \ddot{x}_{v}-l_{p} m_{p} \cos (\theta) \ddot{\theta} & =-u_{1} \sin (\alpha)+D_{x}, \\
-l_{p} m_{p} \cos (\theta) \dot{\theta}^{2}+\left(m_{v}+m_{p}\right)\left(g+\ddot{y}_{v}-l_{p} m_{p} \sin (\theta) \ddot{\theta}\right. & =u_{1} \cos (\alpha)+D_{y} \\
i_{v} \dot{\alpha} & =u_{2}+D_{\alpha}, \\
-l_{p} m_{p} \cos (\theta)\left(\ddot{x}_{v}-l_{p} m_{p} \sin (\theta)\left(g+\left(\ddot{y}_{v}\right)+\left(i_{p}+l_{p}^{2} m_{p}\right) \ddot{\theta}\right.\right. & =0 .
\end{aligned}
$$

Then, the equations in (5a) represent the PVTOL-ASIPL, and they can be expressed in a compact form as

$$
M \ddot{q}+C(q, \dot{q}) \dot{q}+G=U+D,
$$

with

$$
\begin{aligned}
& M=\left[\begin{array}{cccc}
m_{v}+m_{p} & 0 & 0 & -l_{p} m_{p} \cos (\theta) \\
0 & \left(m_{v}+m_{p}\right) & 0 & -l_{p} m_{p} \sin (\theta) \\
0 & 0 & i & 0 \\
-l_{p} m_{p} \cos (\theta) & -l_{p} m_{p} \sin (\theta) & 0 & \left(i_{p}+l_{p}^{2} m_{p}\right)
\end{array}\right], \\
& C=\left[\begin{array}{cccc}
0 & 0 & 0 & l_{p} m_{p} \sin (\theta) \dot{\theta} \\
0 & 0 & 0 & -l_{p} m_{p} \cos (\theta) \dot{\theta} \\
0 & 0 & 0 & 0 \\
0 & 0 & 0 & 0
\end{array}\right] \\
& G=\left[\begin{array}{c}
0 \\
m_{v}+m_{p} \\
0 \\
-l_{p} m_{p} \sin (\theta)
\end{array}\right] \\
& U=\left[\begin{array}{llll}
-u_{1} \sin (\alpha) & u_{1} \cos (\alpha) & u_{2} & 0
\end{array}\right]^{\top} \text {, } \\
& D=\left[\begin{array}{llll}
D_{x} & D_{y} & D_{\alpha} & D_{\theta}
\end{array}\right]^{\top} \text {, }
\end{aligned}
$$

where $M$ is a positive semidefinite matrix, $C$ is the Coriolis matrix, $G$ is the gravity matrix, $U$ is the input control matrix, and $D$ is the external disturbance matrix. The terms $D_{x}, D_{y}$, and $D_{\alpha}$ are assumed to be unmodelled owing to the external disturbances and are defined as follows [30, 31]:

$$
\begin{aligned}
& D_{x}=a_{x} \dot{x}_{v}, \\
& D_{y}=a_{y} \dot{y}_{v}, \\
& D_{\alpha}=a_{\alpha} \dot{\alpha}, \\
& D_{\theta}=0 .
\end{aligned}
$$

Finally, because $M$ is not a singular matrix, it is possible to represent the dynamical model of the PVTOL aircraft system with an inverted pendular load as

$$
\begin{aligned}
\ddot{x}_{v} & =\frac{e_{x 1}+e_{x 2}}{2 m_{v}\left(m_{v}+m_{p}\right)}, \\
\ddot{y}_{v} & =\frac{e_{y 1}+e_{y 2}}{2 m_{v}\left(m_{v}+m_{p}\right)}, \\
\ddot{\alpha} & =u_{2}+a_{\alpha} \dot{\alpha}, \\
\ddot{\theta} & =\frac{-u_{1} \sin (\alpha-\theta)+a_{x} \cos (\theta) \dot{x}_{v}+a_{y} \sin (\theta) \dot{y}_{v}}{l p m},
\end{aligned}
$$


where

$$
\begin{aligned}
& e_{x 1}=-u_{1}\left(\left(2 m_{v}+m_{p}\right) \sin (\alpha)+m_{p} \sin (\alpha-2 \theta)\right), \\
& e_{x 2}=a_{x}\left(2 m_{v}+m_{p}+m_{p} \cos (2 \theta)\right) \dot{x}_{v}+a_{y} m_{p} \sin (2 \theta) \dot{y}_{v}-2 l_{p} m_{v} m_{p} \sin (\theta) \dot{\theta}^{2}, \\
& e_{y 1}=-2 g m_{v}\left(m_{v}+m_{p}\right)+\left(2 m_{v}+m_{p}\right) u_{1} \cos (\alpha)-m_{p} u_{1} \cos (\alpha-2 \theta), \\
& e_{y 2}=a_{x} m_{p} \sin (2 \theta) \dot{x}_{v}+a_{y}\left(2 m_{v}+m_{p}-m_{p} \cos (2 \theta)\right) \dot{y}_{v}+2 l_{p} m_{v} m_{p} \cos (\theta) \dot{\theta}^{2} .
\end{aligned}
$$

2.1. Problem Statement. The control goal consists in proposing an algorithm to accomplish stabilization at the origin of the PVTOL aircraft system with an inverted pendular load. The maneuvers are trajectory tracking tasks involving a step-by-step procedure, consisting of (1) the stabilization of the coordinate $\alpha$; (2) the stabilization of the coordinate $y_{v}$; and (3) further stabilization of coordinates $x_{v}$ and $\theta$, even in the presence of disturbances due to the aerodynamic effects.

We have expressed the system in its minimal representation form, which will allow us to decouple it and simplify it. Instead of working with a complex system, we work with a few simple systems that embody the dynamics of the original one. We are now in a position to design and propose the control scheme in the following section.

\section{Control Scheme}

This section establishes the framework to solve the main control problem. To this end, please consider that the input control $u_{2}$ acts over $\alpha$ plus disturbance $D_{\alpha}$. So, it is possible to design a control law for $\alpha$ using a fictitious controller for the PVTOL aircraft with an inverted pendular load $[9,11]$. Then, through linear transformations, a GPI law is used for the PVTOL takeoff and landing maneuvers. Once the GPI law stabilized the PVTOL aircraft height, a change of coordinates allows expressing the system as a chain of integrators nonlinearly perturbed, allowing to propose the nested saturation function-based stabilizing controller. Figure 2 presents the schematic diagram of the closed-loop system.

3.1. Controlling the PVTOL Aircraft Attitude ( $\alpha)$. It is clear that the third equation (9c) consists of a double-chain integrator with nonlinear disturbance $D_{\alpha}$ and control input $u_{2}$. Then, a control law for tracking trajectory is searched for $\alpha$.

3.1.1. Control Statement. The dynamical equation for the roll attitude angle $(\alpha)$ can be expressed as

$$
\ddot{\alpha}=u_{2}+\xi(t)
$$

where $\xi(t)$ is a lumped generalized disturbance input.

Also, according to Lozano Hernández et al. and Fliess et al. [31, 32], to overcome the lack of available measurements of $\dot{\alpha}$, an integral reconstructor $\left(\widehat{\dot{\alpha}}=\int_{0}^{t} u(\tau) \mathrm{d} \tau\right)$ can be proposed, and using the local approximation of the disturbance input, it is possible to propose the control input $u_{2}$ as

$$
\begin{aligned}
u_{2}= & \ddot{\alpha}_{d}-k_{\alpha_{4}} \dot{e}_{\alpha}-k_{\alpha_{3}} e_{\alpha}-k_{\alpha_{2}} \int_{\tau_{1}=0}^{t} e_{\alpha} \mathrm{d} \tau_{1}-k_{\alpha_{1}} \int_{\tau_{1}=0}^{t} \int_{\tau_{2}=0}^{\tau_{1}} e_{\alpha} \mathrm{d} \tau_{2} \mathrm{~d} \tau_{1} \\
& -k_{\alpha_{0}} \int_{\tau_{1}=0}^{t} \int_{\tau_{2}=0}^{\tau_{1}} \int_{\tau_{3}=0}^{\tau_{2}} e_{\alpha} \mathrm{d} \tau_{3} \mathrm{~d} \tau_{2} \mathrm{~d} \tau_{1},
\end{aligned}
$$

where $\alpha_{d}$ is a smooth reference signal for the state $\alpha$, which is twice differentiable. Also, $\dot{e}_{\alpha}=\widehat{\dot{\alpha}}-\dot{\alpha}_{d}$ and $e_{\alpha}=\alpha-\alpha_{d}$, and the relation between the actual value of $\dot{\alpha}$ and $\hat{\dot{\alpha}}$ is expressed by

$$
\dot{\alpha}=\widehat{\dot{\alpha}}+\int_{0}^{t} \xi(\tau) \mathrm{d} \tau+\dot{\alpha}(0) .
$$

Substituting (12) into (11) and expressing the resulting dynamics in terms of the tracking error, the following dynamics are obtained:

$$
e_{\alpha}^{(5)}+k_{\alpha_{4}} e_{\alpha}^{(4)}+k_{\alpha_{3}} e_{\alpha}^{(3)}+k_{\alpha_{2}} \ddot{e}_{\alpha}+k_{\alpha_{1}} \dot{e}_{\alpha}+k_{\alpha_{0}} e_{\alpha}=0
$$

where $k_{\alpha_{i}}$, with $i=0, \ldots, 4$, are selected such that characteristic polynomial $s^{5}+k_{\alpha_{4}} s^{4}+k_{\alpha_{3}} s^{3}+k_{\alpha_{2}} s^{2}+k_{\alpha_{1}} s+k_{\alpha_{0}}=0$ is Hurwitz, reducing the undesirable effects of the nonlinear disturbances $[33,34]$. So, the dynamic error is exponentially asymptotically stable at the origin. This fact allows using $\alpha_{d}$ as a fictitious control for subsystems (9a), (9b), and (9d). This proposal was previously used in other works dealing with the stabilization of PVTOL aircrafts $[9,12]$.

3.2. Simplified PVTOL Aircraft System with an Inverted Pendular Load. After $\alpha \longrightarrow \alpha_{d}$ and applying the controller $u_{2}$, the following system of equations represents the PVTOL aircraft system with an inverted pendular load:

$$
\begin{aligned}
& \ddot{x}_{v}=\frac{e_{x 1}+e_{x 2}}{2 m_{v}\left(m_{v}+m_{p}\right)}, \\
& \ddot{y}_{v}=\frac{e_{y 1}+e_{y 2}}{2 m_{v}\left(m_{v}+m_{p}\right)},
\end{aligned}
$$




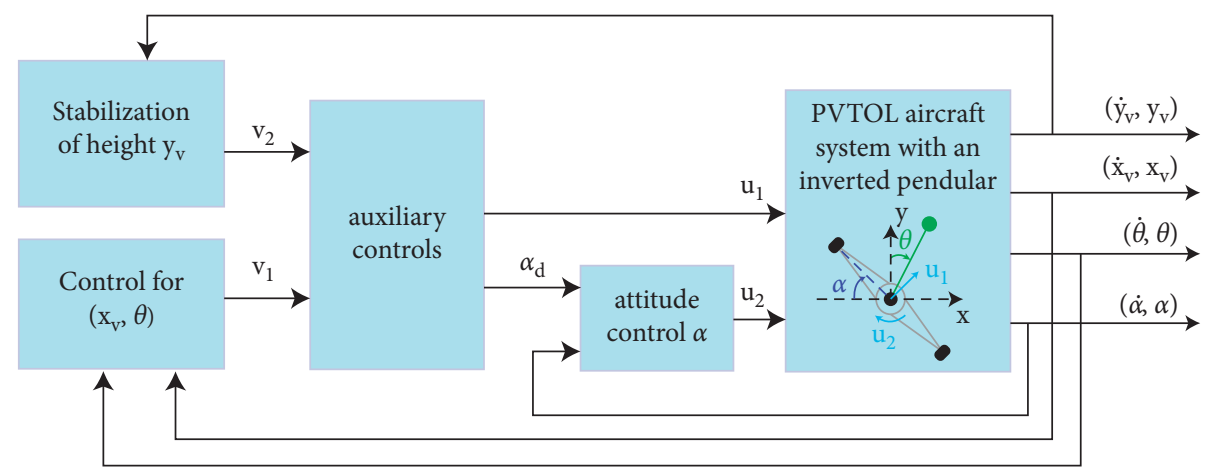

FIgURE 2: Control scheme of the PVTOL aircraft system with an inverted pendular load.

$$
\ddot{\theta}=\frac{-u_{1} \sin \left(\alpha_{d}-\theta\right)+a_{x} \cos (\theta) \dot{x}_{v}+a_{y} \sin (\theta) \dot{y}_{v}}{l p m},
$$

with

$$
\begin{aligned}
& e_{x 1}=-u_{1}\left(\left(2 m_{v}+m_{p}\right) \sin \left(\alpha_{d}\right)+m_{p} \sin \left(\alpha_{d}-2 \theta\right)\right) \\
& e_{x 2}=a_{x}\left(2 m_{v}+m_{p}+m_{p} \cos (2 \theta)\right) \dot{x}_{v}+a_{y} m_{p} \sin (2 \theta) \dot{y}_{v}-2 l_{p} m_{v} m_{p} \sin (\theta) \dot{\theta}^{2} \\
& e_{y 1}=-2 g m_{v}\left(m_{v}+m_{p}\right)+\left(2 m_{v}+m_{p}\right) u_{1} \cos \left(\alpha_{d}\right)-m_{p} u_{1} \cos \left(\alpha_{d}-2 \theta\right) \\
& e_{y 2}=a_{x} m_{p} \sin (2 \theta) \dot{x}_{v}+a_{y}\left(2 m_{v}+m_{p}-m_{p} \cos (2 \theta)\right) \dot{y}_{v}+2 l_{p} m_{v} m_{p} \cos (\theta) \dot{\theta}^{2}
\end{aligned}
$$

where $\alpha_{d}$ and $u_{1}$ are the input controls.

Thus, we propose the following control laws [12]:

$$
x_{v}=\frac{\hat{x}_{v}}{2 m_{v}\left(m_{v}+m_{p}\right)},
$$

$$
\begin{aligned}
& u_{1}=\sqrt{r_{1}^{2}+r_{2}^{2}}, \\
& \alpha_{d}=\arctan \left(\frac{r_{2}}{r_{1}}\right),
\end{aligned}
$$

where $r_{1}>0$ and $r_{2}>0$ are auxiliary control inputs.

To obtain the dynamic model, the following change of coordinates is applied to model (15a)-(15c):

$$
\begin{aligned}
y_{v} & =\frac{\widehat{y}_{v}}{2 m_{v}\left(m_{v}+m_{p}\right)}, \\
\theta & =\frac{\widehat{\theta}}{l_{p} m_{v}} .
\end{aligned}
$$

Therefore, models (15a)-(15c) transform into the following system:

$$
\begin{aligned}
\ddot{\hat{x}}_{v}= & -\left(2 m_{v}+m_{p}\right)\left(r_{2}-a_{x} \dot{x}_{v}\right)+m_{p} \cos (2 \theta)\left(-r_{2}+a_{x} \dot{\hat{x}}_{v}\right) \\
& +m_{p} \sin (2 \theta)\left(r_{1}+a_{y} \dot{\hat{y}}_{v}\right)-2 l_{p} m_{v} m_{p} \sin (\theta) \dot{\theta}^{2}, \\
\ddot{\hat{y}}_{v}= & -2 g m_{v}\left(m_{v}+m_{p}\right)+\left(2 m_{v}+m_{p}\right) r_{1}-m_{p}\left(r_{1} \cos (2 \theta)+r_{2} \sin (2 \theta)\right), \\
& +a_{x} m_{p} \sin (2 \theta) \dot{\hat{x}}_{v}+a_{y}\left(2 m_{v}+m_{p}-m_{p} \cos (2 \theta)\right) \dot{\hat{y}}_{v}+2 l_{p} m_{v} m_{p} \cos (\theta) \dot{\theta}^{2} \\
\ddot{\hat{\theta}}= & \cos (\theta)\left(-r_{2}+a_{x} \dot{\hat{x}}_{v}\right)+\sin (\theta)\left(r_{1}+a_{y} \dot{\hat{y}}_{v}\right) .
\end{aligned}
$$


Hence, system (19) takes the compact form

$$
\left[\begin{array}{l}
\ddot{\widehat{x}}_{v} \\
\ddot{\hat{y}}_{v}
\end{array}\right]=M_{2}\left[\begin{array}{l}
r_{1} \\
r_{2}
\end{array}\right]+C_{2},
$$

$$
\begin{aligned}
M_{2} & =\left[\begin{array}{cc}
m_{p} \sin (2 \theta) & -\left(2 m_{v}+m_{p}+m_{p} \cos (2 \theta)\right) \\
\left(2 m_{v}+m_{p}\right)-m_{p}(\cos (2 \theta)) & -m_{p}(\sin (2 \theta))
\end{array}\right], \\
C_{2} & =\left[\begin{array}{c}
-2 l_{p} m_{v} m_{p} \sin (\theta) \dot{\theta}^{2} \\
-2 g m_{v}\left(m_{v}+m_{p}\right)+2 l_{p} m_{v} m_{p} \cos (\theta) \dot{\theta}^{2}-2 g m_{v}\left(m_{v}+m p\right)
\end{array}\right] .
\end{aligned}
$$

Then, given system (19) and ignoring the effect of the disturbances, the following partial feedback control can be proposed $[1,9]$ :

$$
\left[\begin{array}{l}
r_{1} \\
r_{2}
\end{array}\right]=M_{2}^{-1}\left(-C_{2}+\left[\begin{array}{l}
v_{1} \\
v_{2}
\end{array}\right]\right),
$$

where $v_{1}$ and $v_{2}$ are new auxiliary control inputs. Thus, the system defined by equation (19), in a closed loop with control model (23), leads us to obtain the following system:

$$
\begin{aligned}
& \ddot{\hat{x}}_{v}=v_{1}+a_{x}\left(2 m_{v}+m_{p}+m_{p} \cos (2 \theta)\right) \dot{\hat{x}}_{v}+a_{y} m_{p} \sin (2 \theta) \dot{\hat{y}}_{v}, \\
& \ddot{\hat{y}}_{v}=v_{2}+a_{x} m_{p} \sin (2 \theta) \dot{\hat{x}}_{v}+a_{y}\left(2 m_{v}+m_{p}-m_{p} \cos (2 \theta)\right) \dot{\hat{y}}_{v}, \\
& \ddot{\theta}=\frac{\cos (\theta)\left(v_{1}+2 a_{x}\left(m_{v}+m_{p}\right) \dot{\hat{x}}\right)+\sin (\theta)\left(2 g m_{v}\left(m_{v}+m_{p}\right)+v_{2}+2 a_{y}\left(m_{v}+m_{p}\right) \dot{\hat{y}}\right)}{2\left(m_{v}+m_{p}\right)} .
\end{aligned}
$$

Notice that the above system is the reduced model for the PVTOL aircraft system with an inverted pendular load, with $v_{1}$ and $v_{2}$ as the control inputs.

3.3. Stabilization of Height $y_{v}$. A GPI controller with a saturation function is applied to obtain the height position control, allowing the tracking control to accomplish the takeoff and landing of the PVTOL aircraft system with an inverted pendular load.

3.3.1. Control Statement. Consider the vertical displacement $y_{v}$ described by (25), and let $v_{2}$ be the control input defined as (26):

$$
\begin{aligned}
v_{2}= & \sigma_{a}\left(\ddot{y}_{d}-k_{y_{4}} \dot{e}_{y}-k_{y_{3}} e_{y}-k_{y_{2}} \int_{\tau_{1}=0}^{t} e_{y} \mathrm{~d} \tau_{1}-k_{y_{1}} \int_{\tau_{1}=0}^{t} \int_{\tau_{2}=0}^{\tau_{1}} e_{y} \mathrm{~d} \tau_{2} \mathrm{~d} \tau_{1}\right. \\
& \left.-k_{y_{0}} \int_{\tau_{1}=0}^{t} \int_{\tau_{2}=0}^{\tau_{1}} \int_{\tau_{3}=0}^{\tau_{2}} e_{y} \mathrm{~d} \tau_{3} \mathrm{~d} \tau_{2} \mathrm{~d} \tau_{1}\right)=\sigma_{a}\left(u_{g p i}\right)
\end{aligned}
$$


where $y_{d}$ is a smooth reference signal twice derivable and $e_{y}=y_{d}-\hat{y}_{v}$.

Also, $\sigma_{L}(q)$ is a saturation function defined as

$$
\sigma_{a}(q)= \begin{cases}\text { if } q>a, & a, \\ \text { if } q<-a, & -a, \\ \text { if }-a<q<a, & q .\end{cases}
$$

Thus, the tracking trajectory error $e_{y}$ is exponentially asymptotically stable.

Proof. The proof is split into two parts. First, it is proven that a saturation function in finite time bounds the error dynamics $e_{y}$. Then, we demonstrate exponentially asymptotic stability.

3.3.2. Error Bounded. The tracking trajectory error is defined as

$$
e_{y}=y_{d}-\widehat{y}_{v}
$$

where $y_{d}$ is the desired height position.

Let us define the following state variables as

$$
e_{1}=e_{y} ; e_{2}=\dot{e}_{y}
$$

Therefore, the dynamic error is transformed into the following system:

$$
\begin{aligned}
\dot{e}_{1}= & e_{2}, \\
\dot{e}_{2}= & y_{d}-v_{2}-a_{x} m_{p} \sin (2 \theta) \dot{\hat{x}}_{v} \\
& -a_{y}\left(2 m_{v}+m_{p}-m_{p} \cos (2 \theta)\right) \dot{\hat{y}}_{v},
\end{aligned}
$$

where $v_{2}$ is the control input.

Now, to use the second method of Lyapunov, consider the following candidate function:

$$
V_{y}=\frac{e_{1}^{2}}{2}+\frac{e_{2}^{2}}{2},
$$

which is positive definite, with time derivative

$$
\dot{V}_{y}=e_{1} e_{2}+e_{2} \dot{e}_{2}=e_{1} e_{2}+e_{2}\left(\ddot{y}_{d}-\sigma_{a}\left(u_{g p i}\right)-a_{x} m_{p} \sin (2 \theta) \dot{\hat{x}}_{v}-a_{y}\left(2 m_{v}+m_{p}-m_{p} \cos (2 \theta)\right) \dot{\hat{y}}_{v}\right) .
$$

Thus, the first and second time derivatives of $y_{d}$ are known and bounded as $\left|M_{y_{d}}\right|$. Besides, in a close neighbourhood where $e_{1}$ and $e_{2}$ are such that $\left|e_{1}\right| \leq \delta_{e_{1}}$ and $\left|e_{2}\right| \leq \delta_{e_{2}}$, the saturation function fulfills $s \sigma_{a}(s) \leqslant 0$. So, parameters were designed as $k_{y_{i}}$ in $u_{g p i}$ such that $e_{2}$ is dominant, and $e_{2} \sigma_{a}\left(u_{g p i}\right)>0$. Finally, the last terms of equation (31) satisfy the following:

$$
\begin{gathered}
\left|a_{x} m_{p} \sin (2 \theta)\right| \leq \delta_{x}, \\
\left|a_{y}\left(2 m_{v}+m_{p}-m_{p} \cos (2 \theta)\right)\right| \leq \delta_{y} .
\end{gathered}
$$

Therefore, computing the time derivative of the candidate Lyapunov function leads to

$$
\dot{V}_{y}=\delta_{e_{1}} \delta_{e_{2}}+\delta_{e_{2}} M_{y_{d}}-e_{2} \sigma_{a}\left(u_{g p i}\right)-\delta_{e_{2}} \delta_{x} \dot{\hat{x}}_{v}-\delta_{e_{2}} \delta_{y} \dot{\hat{y}}_{v},
$$

and if $\dot{\hat{x}}_{v}$ and $\dot{\hat{y}}_{v}$ are small enough such that $e_{2} \sigma_{a}\left(u_{g p i}\right)>\delta_{e_{1}} \delta_{e_{2}}+\delta_{e_{2}} M_{y_{d}}-\delta_{e_{2}} \delta_{x} \dot{\hat{x}}_{v}-\delta_{e_{2}} \delta_{y} \dot{\hat{y}}_{v}$, then $\dot{V}_{y}<0$ and $\hat{y}_{v}$ are bounded in finite time. Thus, after time $T$, when this condition is satisfied, control law (26) takes the following structure:

$$
\begin{aligned}
v_{2}= & y_{d}-k_{y_{4}} \dot{e}_{y}-k_{y_{3}} e_{y}-k_{y_{2}} \int_{\tau_{1}=0}^{t} e_{y} \mathrm{~d} \tau_{1}-k_{y_{1}} \int_{\tau_{1}=0}^{t} \int_{\tau_{2}=0}^{\tau_{1}} e_{y} \mathrm{~d} \tau_{2} \mathrm{~d} \tau_{1} \\
& -k_{y_{0}} \int_{\tau_{1}=0}^{t} \int_{\tau_{2}=0}^{\tau_{1}} \int_{\tau_{3}=0}^{\tau_{2}} e_{y} \mathrm{~d} \tau_{3} \mathrm{~d} \tau_{2} \mathrm{~d} \tau_{1} .
\end{aligned}
$$

From the above, equation (24b) is expressed as

$$
\ddot{\widehat{y}}_{v}=v_{2}+\xi_{1}(t)
$$

where $\xi_{1}(t)$ is a lumped generalized disturbance input.

Then, to overcome the lack of available measurements of $\dot{\hat{y}}_{v}$, the following integral reconstructor is introduced [31]:

$$
\overline{\hat{\hat{y}}}_{v}=\int_{0}^{t} v_{2}(\tau) \mathrm{d} \tau \text {. }
$$

Using the local approximation of the disturbance input, the relation between the actual value of $\dot{\hat{y}}_{v}$ and $\overline{\hat{\hat{y}}}_{v}$ is expressed by

$$
\dot{\hat{y}}_{v}=\overline{\hat{y}}_{v}+\int_{0}^{t} \xi(\tau) \mathrm{d} \tau+\dot{\hat{y}}_{v}(0)
$$

Now, differentiating equation (38) and substituting into (36), the control law $v_{2}$ takes the form 


$$
\begin{aligned}
v_{2}= & \ddot{y}_{d}-k_{y_{4}} \dot{\bar{e}}_{y}-k_{y_{3}} \bar{e}_{y}-k_{y_{2}} \int_{\tau_{1}=0}^{t} \bar{e}_{y} \mathrm{~d} \tau_{1}-k_{y_{1}} \int_{\tau_{1}=0}^{t} \int_{\tau_{2}=0}^{\tau_{1}} \bar{e}_{y} \mathrm{~d} \tau_{2} \mathrm{~d} \tau_{1} \\
& -k_{y_{0}} \int_{\tau_{1}=0}^{t} \int_{\tau_{2}=0}^{\tau_{1}} \int_{\tau_{3}=0}^{\tau_{2}} \bar{e}_{y} \mathrm{~d} \tau_{3} \mathrm{~d} \tau_{2} \mathrm{~d} \tau_{1},
\end{aligned}
$$

with $\bar{e}_{y}=y_{v}-y_{d}$ and $\dot{\bar{e}}_{y}=\overline{\hat{\hat{y}}}_{v}-\dot{y}_{d}$

Substituting (39) into (36) is possible to express the dynamic error $\bar{e}_{y}$ as follows:

$$
\bar{e}_{y}^{(5)}+k_{y_{4}} \bar{e}_{y}^{(4)}+k_{y_{3}} \bar{e}_{y}^{(3)}+k_{y_{2}} \ddot{\bar{e}}_{y}+k_{y_{1}} \dot{\bar{e}}_{y}+k_{y_{0}} \bar{e}_{y}=0 .
$$

The coefficients $k_{y_{4}}, k_{y_{3}}, k_{y_{2}}, k_{y_{1}}$, and $k_{y_{0}}$ are selected such that the polynomial $S^{(5)}+k_{y_{4}} S^{(4)}+k_{y_{3}} S^{(3)}+k_{y_{2}} S^{2}+k_{y_{1}} S+k_{y_{0}} \quad$ is Hurwitz, eliminating the nonlinear disturbances [33]. Then, tracking trajectory error $\bar{e}_{y}$ is exponentially asymptotically stable to zero.

3.4. Control for $\left(x_{v}, \theta\right)$. This section proposes a nested saturation-based controller strategy to stabilize the PVTOLASIPL horizontal position and roll angle. Notice that the nested saturation-based controller strategy allows stabilizing nonlinear systems that can be approximately expressed as a chain of integrators [35-37]. Thus, our stability problem will be solved as follows. First, we introduce a linear transformation for the stabilizing controller. Then, we demonstrate that the proposed controller guarantees boundedness and converges to zero, in finite time, of the whole state.

3.5. Expressing the PVTOL-ASIPL as a Chain of Integrators. After the application of the controller $v_{2}$, system (25) can be reduced to the subsystem $\left(\widehat{x}_{v}, \theta\right)$ as follows:

$$
\begin{aligned}
& \ddot{\widehat{x}}_{v}=v_{1}+a_{x}\left(2 m_{v}+m_{p}+m_{p} \cos (2 \theta)\right) \dot{\hat{x}}_{v}, \\
& \ddot{\theta}=\frac{2 g m_{v}\left(m_{v}+m p\right) \sin (\theta)+\cos (\theta)\left(v_{1}+2 a_{x}\left(m_{v}+m_{p}\right) \dot{\hat{x}}_{v}\right)}{2\left(m_{v}+m_{p}\right)} .
\end{aligned}
$$

Then, introduce the following coordinates' change:

$$
\begin{aligned}
\widehat{x}_{v} & =x_{v}\left(-2 g m_{v}\left(m_{v}+m_{p}\right)\right), \\
\widehat{\theta} & =\theta\left(g m_{v}\right), \\
v_{1} & =2\left(m_{v}+m_{p}\right)\left(g m_{v}(v \sec (\theta)-\tan (\theta))-a_{x} \dot{x}_{v}\right) .
\end{aligned}
$$

System (42) takes the following form:

$$
\begin{aligned}
\ddot{x}_{v} & =v \sec (\theta)-\tan (\theta)-\epsilon_{x} \sin (\theta)^{2} \dot{x}_{v}, \\
\ddot{\theta} & =v,
\end{aligned}
$$

where $\epsilon_{x} \sin (\theta)^{2} \dot{x}_{v}$ is considered as a nonlinear disturbance and $\epsilon_{x}$ is an unknown constant that depends on variables $m_{v}, m_{p}, g$, and $a_{x}$ (Note that $\epsilon_{x}=\left(m_{p} / g * m_{v} *\left(m_{v}+\right.\right.$ $\left.\left.\left.m_{p}\right)\right) a_{x}.\right)$. Notice that subsystem (43) is similar to the cartpole system plus a nonlinear disturbance [38].
3.5.1. Control Statement. Based on work [3], we define the following state variables $x_{1}=x_{v}, x_{2}=\dot{x}_{v}, \theta_{1}=\theta$, and $\theta_{2}=\dot{\theta}$. Then, we express the dynamic system as

$$
\begin{aligned}
& \dot{x}_{1}=x_{2}, \\
& \dot{x}_{2}=-v \sec \left(\theta_{1}\right)+\tan \left(\theta_{1}\right)+\epsilon_{x} \sin \left(\theta_{1}\right)^{2} x_{2}, \\
& \dot{\theta}_{1}=\theta_{2}, \\
& \dot{\theta}_{1}=v .
\end{aligned}
$$

To express system (44) as a chain of integrators with a nonlinear perturbation and propose a controller for the stabilization of the subsystem $\left(x_{v}, \theta\right)$, applying the decoupling theorem [3, 39], the following global nonlinear transformation is introduced:

$$
\begin{aligned}
& z_{1}=\frac{1+\tan \left(\theta_{1} / 2\right)}{1-\tan \left(\theta_{1} / 2\right)}+x_{1}, \\
& z_{2}=\frac{\theta_{2}}{\cos \left(\theta_{1}\right)+x_{2}}, \\
& \omega_{1}=\tan \left(\theta_{1}\right), \\
& \omega_{2}=\theta_{2} \sec ^{2}\left(\theta_{1}\right), \\
& v_{f}=\sec ^{2}\left(\theta_{1}\right) v+2 \theta_{2}^{2} \tan \left(\theta_{1}\right) \sec ^{2}\left(\theta_{1}\right) .
\end{aligned}
$$

Hence, the transformed system into a chain of integrators is given by

$$
\begin{aligned}
& \dot{z}_{1}=z_{2}, \\
& \dot{z}_{2}=\omega_{1}+\frac{\omega_{1} \omega_{1}^{2}}{\left(1+\omega_{1}^{2}\right)}+\frac{\epsilon_{x} \omega_{2} \omega_{1}^{2}}{\left(1+\omega_{1}^{2}\right)^{3 / 2}}-\frac{\epsilon_{x} z_{2} \omega_{1}^{2}}{1+\omega_{1}^{2}}, \\
& \dot{\omega}_{1}=\omega_{2}, \\
& \dot{\omega}_{2}=v_{f} .
\end{aligned}
$$

3.6. Nested Saturation Function-Based Controller. Inspired by Teel [40], a linear transformation is proposed to obtain the stabilizing controller for system (46) as follows:

$$
\left[\begin{array}{l}
q_{1} \\
q_{2} \\
q_{3} \\
q_{4}
\end{array}\right]=\left[\begin{array}{llll}
1 & 3 & 3 & 1 \\
0 & 1 & 2 & 1 \\
0 & 0 & 1 & 1 \\
0 & 0 & 0 & 1
\end{array}\right]\left[\begin{array}{l}
z_{1} \\
z_{2} \\
\omega_{1} \\
\omega_{2}
\end{array}\right] .
$$




$$
\begin{aligned}
\dot{q}_{1}= & v_{f}+q_{2}+q_{3}+q_{4}+3 G_{1}\left(q_{3}-q_{4}\right) q_{4}^{2} \\
& +3 G_{2}\left(q_{3}-q_{4}\right) q_{4} \epsilon_{x}+3 G_{3}\left(q_{3}-q_{4}\right)\left(q_{2}-2 q_{3}+q_{4}\right) \epsilon_{x}, \\
\dot{q}_{2}= & v_{f}+q_{3}+q_{4}+G_{1}\left(q_{3}-q_{4}\right) q_{4}^{2} \\
& +G_{2}\left(q_{3}-q_{4}\right) q_{4} \epsilon_{x}+G_{3}\left(q_{3}-q_{4}\right)\left(q_{2}-2 q_{3}+q_{4}\right) \epsilon_{x}, \\
\dot{q}_{3}= & v_{f}+q_{4}, \\
\dot{q}_{4}= & v_{f},
\end{aligned}
$$

for which the following nested saturation function-based stabilizing controller is proposed:

$$
v_{f}=-\sigma_{c_{1}}\left(q_{4}+\sigma_{c_{2}}\left(q_{3}+\sigma_{c_{3}}\left(q_{2}+\sigma_{c_{4}}\left(q_{1}\right)\right)\right)\right),
$$

where $\sigma_{c_{i}}$ is defined by equation (26), and $G_{i}$ with $i=1,2,3$ is given by

$$
\begin{aligned}
& G_{1}(s)=\frac{s}{\left(1+s^{2}\right)^{3 / 2}}, \\
& G_{2}(s)=\frac{s^{2}}{\left(1+s^{2}\right)^{3 / 2}}, \\
& G_{3}(s)=\frac{s^{2}}{1+s^{2}} .
\end{aligned}
$$

Remark 1. Note that $\max \left(G_{1}(s)\right)=K_{1}=2 /(3 \sqrt{3})$ when $s=$ $1 / \sqrt{2}$ and $\min \left(G_{1}(s)\right)=-2 /(3 \sqrt{3})$ when $s=-1 / \sqrt{2}$ and $\lim _{s \longrightarrow \text { inf }} G_{1}(s)=0$. Besides, $\max \left(G_{2}(s)\right)=K_{2}=2 /(3 \sqrt{3})$ when $s= \pm \sqrt{2}$ and $\min \left(G_{1}(s)\right)=0$ when $s=0$ and $\lim _{s \longrightarrow \inf } G_{2}(s)=0$. Additionally, $\min \left(G_{3}(s)\right)=0$ when $s=$ 0 and $\lim _{s \longrightarrow \mathrm{inf}} G_{3}(s)=K_{3}=1$.

3.7. Whole State Boundedness. Now, we prove that the proposed controller (49) ensures whole state boundedness. Moreover, the bound of each state directly depends on the designed parameters of the controller.

Step 1. A positive definite function is defined as

$$
V_{4}=\frac{q_{4}^{2}}{2} \text {. }
$$

The time derivative of $V_{4}$ is expressed by

$$
\dot{V}_{4}=q_{4} \dot{q}_{4}=q_{4} v_{f}=-q_{4} \sigma_{c_{1}}\left(q_{4}+\sigma_{c_{2}}\left(q_{3}+\sigma_{c_{3}}\left(q_{2}+\sigma_{c_{4}}\left(q_{1}\right)\right)\right)\right) \text {, }
$$

where $c_{1}$ and $c_{2}$ are selected such that $c_{1}>2 c_{2}$. It is clear that $\dot{V}_{4}<0$ when $\left|q_{4}\right| \geq c_{2}$; therefore, there exists a finite time $T_{1}>0$ such that

$$
\left|q_{4}\right| \leq c_{2}, \quad \forall t>T_{1} .
$$

Thus, when this condition is satisfied, the control law (49) takes the following structure:

$$
v_{f}=-q_{4}-\sigma_{c_{2}}\left(q_{3}+\sigma_{c_{3}}\left(q_{2}+\sigma_{c_{4}}\left(q_{1}\right)\right)\right) .
$$

Step 2. Behaviour analysis of $q_{3}$ : let us consider the following positive definite function:

$$
V_{3}=\frac{q_{3}^{2}}{2} .
$$

Differentiating it with respect to time and after substituting (54) into $\dot{q}_{3}$, the following expression is obtained:

$$
\dot{V}_{3}=q_{3} \dot{q}_{3}=q_{3}\left(v_{f}+q_{4}\right)=-q_{3} \sigma_{c_{2}}\left(q_{3}+\sigma_{c_{3}}\left(q_{2}+\sigma_{c_{4}}\left(q_{1}\right)\right)\right) \text {. }
$$

To ensure that $\dot{V}_{3}<0$ is achieved, the following conditions must be satisfied:

$$
c_{2}>2 c_{3}, \quad\left|q_{3}\right| \geq c_{3} .
$$

Then, there exists a finite time $T_{2}>T_{1}$ after which

$$
\left|q_{3}\right| \leq c_{3}, \quad \forall t>T_{2} .
$$

Therefore, $q_{3}$ is bounded, and the control law takes the following form:

$$
v_{f}=-q_{4}-q_{3}-\sigma_{c_{3}}\left(q_{2}+\sigma_{c_{4}}\left(q_{1}\right)\right) .
$$

Step 3. The following positive definite function is introduced:

$$
V_{2}=\frac{q_{2}^{2}}{2} .
$$

Differentiating $V_{2}$ and after substituting (60) into the second equation of (48), the following is obtained:

$$
\begin{aligned}
\dot{V}_{2}= & q_{2} \dot{q}_{2}=-q_{2}\left[\sigma_{c_{3}}\left(q_{2}+\sigma_{c_{4}}\left(q_{1}\right)\right)-G_{1}\left(q_{3}-q_{4}\right) q_{4}^{2}\right. \\
& \left.-G_{2}\left(q_{3}-q_{4}\right) q_{4} \epsilon_{x}-G_{3}\left(q_{3}-q_{4}\right)\left(q_{2}-2 q_{3}+q_{4}\right)\right],
\end{aligned}
$$

where $c_{3}$ and $c_{2}$ must satisfy the relation $c_{3}>2 c_{4}+G_{1}\left(q_{3}-q_{4}\right)_{4}^{2}+G_{2}\left(q_{3}-q_{4}\right) q_{4} \epsilon_{x}+G_{3}\left(q_{3}-\right.$

$\left.q_{4}\right)\left(q_{2}-2 q_{3}+q_{4}\right)$. It is easy to observe that if $\left|q_{2}\right|>c_{4}+G_{2}\left(q_{3}-q_{4}\right) q_{4} \epsilon_{x}+G_{3}\left(q_{3}-q_{4}\right)\left(q_{2}-2 q_{3}+q_{4}\right)$,

$V_{2}<0$. Hence, there exists a finite time $T_{3}>T_{2}$ after which

$$
\begin{aligned}
\left|q_{2}\right| \leq & c_{4}+G_{2}\left(q_{3}-q_{4}\right) q_{4} \epsilon_{x} \\
& +G_{3}\left(q_{3}-q_{4}\right)\left(q_{2}-2 q_{3}+q_{4}\right) ; \quad \forall t>T_{3} .
\end{aligned}
$$
to be

Therefore, $q_{2}$ is bounded, and the control $v_{f}$ is revealed

$$
v_{f}=-q_{4}-q_{3}-q_{2}-\sigma_{c_{4}}\left(q_{1}\right) .
$$

Step 4. Substituting (63) into the first equation of (48), we obtain 


$$
\begin{aligned}
\dot{q}_{1}= & -\sigma_{c_{4}}\left(q_{1}\right)+3 G_{1}\left(q_{3}-q_{4}\right) q_{4}^{2}+3 G_{2}\left(q_{3}-q_{4}\right) q_{4} \epsilon_{x} \\
& +3 G_{3}\left(q_{3}-q_{4}\right)\left(q_{2}-2 q_{3}+q_{4}\right) \epsilon_{x} .
\end{aligned}
$$

To demonstrate that $q_{1}$ is bounded, a positive definite function is defined as follows:

$$
V_{1}=\frac{q_{1}^{2}}{2}
$$

Differentiating $V_{1}$ along the trajectories of (66) leads to

$$
\begin{aligned}
\dot{V}_{1}= & q_{1} \dot{q}_{1}=-q_{1}\left(\sigma_{c_{4}}\left(q_{1}\right)-3 G_{1}\left(q_{3}-q_{4}\right) q_{4}^{2}\right. \\
& \left.-3 G_{2}\left(q_{3}-q_{4}\right) q_{4} \epsilon_{x}-3 G_{3}\left(q_{3}-q_{4}\right)\left(q_{2}-2 q_{3}+q_{4}\right)\right),
\end{aligned}
$$

where $c_{4}$ must be selected so that $c_{4}>3 G_{1}\left(q_{3}-q_{4}\right) q_{4}^{2}+$ $3 G_{2}\left(q_{3}-q_{4}\right) q_{4} \epsilon_{x}+3 G_{3}\left(q_{3}-q_{4}\right)\left(q_{2}-2 q_{3}+q_{4}\right) \quad$ and $\left|q_{1}\right|>3 G_{1}\left(q_{3}-q_{4}\right) q_{4}^{2}+3 G_{2}\left(q_{3}-q_{4}\right) \quad q_{4} \epsilon_{x}+3 G_{3}\left(q_{3}-q_{4}\right)$ $\left(q_{2}-2 q_{3}+q_{4}\right)$ to achieve $V_{1}<0$. Therefore, there exists a finite time $T_{4}>T_{3}$ such that

$$
\left|q_{1}\right|<3 G_{1}\left(q_{3}-q_{4}\right) q_{4}^{2}+3 G_{2}\left(q_{3}-q_{4}\right) q_{4} \epsilon_{x}+3 G_{3}\left(q_{3}-q_{4}\right)\left(q_{2}-2 q_{3}+q_{4}\right), \quad \forall t>T_{4} .
$$

Consequently, $q_{1}$ is also bounded. Finally, the values of parameters $c_{1}, c_{2}, c_{3}$, and $c_{4}$ can be determined by the following inequalities:

$$
\begin{aligned}
& c_{1}>2 c_{2} \text {, } \\
& c_{2}>2 c_{3} \text {, } \\
& c_{3}>2 c_{4}+G_{1}\left(q_{3}-q_{4}\right) q_{4}^{2}+G_{2}\left(q_{3}-q_{4}\right) q_{4} \epsilon_{x}+G_{3}\left(q_{3}-q_{4}\right)\left(q_{2}-2 q_{3}+q_{4}\right) \text {, } \\
& c_{1}=28 r \text {, } \\
& c_{2}=14 r \text {, } \\
& c_{3}=7 r \text {, } \\
& c_{4}=3 r \text {, }
\end{aligned}
$$$$
c_{4}>3 G_{1}\left(q_{3}-q_{4}\right) q_{4}^{2}+3 G_{2}\left(q_{3}-q_{4}\right) q_{4} \epsilon_{x}+3 G_{3}\left(q_{3}-q_{4}\right)\left(q_{2}-2 q_{3}+q_{4}\right) \text {. }
$$

From the above conditions, the set of control parameters can be selected as

where $r$ is directly related to the magnitude of the system disturbances.

3.8. Whole State Convergence to Zero. Here, we prove that the closed-loop system, provided by (48) and (49) and satisfying (70), is asymptotically stable.

Notice that, after $t>T_{4}$, the control law (49) is no longer saturated; that is,

$$
v_{f}=-q_{4}-q_{3}-q_{2}-q_{1} \text {, }
$$

and the closed-loop system turns out to be

$$
\begin{aligned}
\dot{q}_{1}= & -q_{1}+3 G_{1}\left(q_{3}-q_{4}\right) q_{4}^{2} \\
& +3 G_{2}\left(q_{3}-q_{4}\right) q_{4} \epsilon_{x}+3 G_{3}\left(q_{3}-q_{4}\right)\left(q_{2}-2 q_{3}+q_{4}\right) \epsilon_{x}, \\
\dot{q}_{2}= & -q_{1}-q_{2}+G_{1}\left(q_{3}-q_{4}\right) q_{4}^{2} \\
& +G_{2}\left(q_{3}-q_{4}\right) q_{4} \epsilon_{x}+G_{3}\left(q_{3}-q_{4}\right)\left(q_{2}-2 q_{3}+q_{4}\right) \epsilon_{x}, \\
\dot{q}_{3}= & -q_{1}-q_{2}-q_{3}, \\
\dot{q}_{4}= & -q_{1}-q_{2}-q_{3}-q_{4} .
\end{aligned}
$$

To demonstrate whole state convergence to zero, the following Lyapunov function is used:

$$
V=\frac{1}{2} q^{\top} q
$$

where $q=\left[q_{1}, q_{2}, \ldots, q_{3}, q_{2}\right]^{\top}$, and differentiating $V$ along the trajectories of equation (72), it is obtained that

$$
\begin{aligned}
\dot{V}= & -q^{\top} M q+\left(3 q_{1}+q_{2}\right)\left(G_{1}\left(q_{3}-q_{4}\right) q_{4}^{2}\right. \\
& \left.+G_{2}\left(q_{3}-q_{4}\right) q_{4} \epsilon_{x}+G_{3}\left(q_{3}-q_{4}\right)\left(q_{2}-2 q_{3}+q_{4}\right) \epsilon_{x}\right),
\end{aligned}
$$

and 


$$
M=\left[\begin{array}{llll}
1 & \frac{1}{2} & \frac{1}{2} & \frac{1}{2} \\
\frac{1}{2} & 1 & \frac{1}{2} & \frac{1}{2} \\
\frac{1}{2} & \frac{1}{2} & 1 & \frac{1}{2} \\
\frac{1}{2} & \frac{1}{2} & \frac{1}{2} & 1
\end{array}\right],
$$

with $M$ being positive definite with $\lambda_{\min }(M) 1 / 2$.

From Remark 1, it is shown that the following relations are satisfied:

$$
\begin{gathered}
\left|\left(3 q_{1}+q_{2}\right) G_{1}\left(q_{3}-q_{4}\right) q_{4}^{2}\right|<\frac{K_{1}}{2}\left|\left(3 q_{1}+q_{2}\right) q_{4}^{2}\right|<\frac{K_{1}}{2}\left(\left(3 q_{1}+q_{2}\right)^{2}+q_{4}^{4}\right), \\
\left|\left(3 q_{1}+q_{2}\right) G_{2}\left(q_{3}-q_{4}\right) q_{4} \epsilon_{x}\right|<\frac{K_{2} \epsilon_{x}}{2}\left|\left(3 q_{1}+q_{2}\right) q_{4}\right|<\frac{K_{2} \epsilon_{x}}{2}\left(\left(3 q_{1}+q_{2}\right)^{2}+q_{4}^{2}\right), \\
\left|\left(3 q_{1}+q_{2}\right) G_{3}\left(q_{3}-q_{4}\right) q_{2} \epsilon_{x}\right|<\frac{K_{3} \epsilon_{x}}{2}\left|\left(3 q_{1}+q_{2}\right) q_{2}\right|<\frac{K_{3} \epsilon_{x}}{2}\left(\left(3 q_{1}+q_{2}\right)^{2}+q_{2}^{2}\right), \\
\left|\left(3 q_{1}+q_{2}\right) G_{3}\left(q_{3}-q_{4}\right) q_{4} \epsilon_{x}\right|<\frac{K_{3} \epsilon_{x}}{2}\left|\left(3 q_{1}+q_{2}\right) q_{4}\right|<\frac{K_{3} \epsilon_{x}}{2}\left(\left(3 q_{1}+q_{2}\right)^{2}+q_{4}^{2}\right) .
\end{gathered}
$$

So, $\dot{V}$ fulfills

$$
\begin{aligned}
\dot{V}< & -\frac{1}{2}\left[q_{1}^{2}-K_{1}\left(3 q_{1}+q_{2}\right)^{2}-K_{2} \epsilon_{x}\left(3 q_{1}+q_{2}\right)^{2}-2 K_{3} \epsilon_{x}\left(3 q_{1}+q_{2}\right)^{2}\right] \\
& -\frac{1}{2} q_{4}^{2}\left[1-K_{1} q_{4}^{2}-K_{3} \epsilon_{x}\right]-\frac{1}{2} q_{2}^{2}\left[1-K_{2} \epsilon_{x}\right] .
\end{aligned}
$$

Hence, the previous inequality is strictly negative definite, and the following constraints are obtained:

$$
\begin{array}{r}
q_{1}^{2}-K_{1}\left(3 q_{1}+q_{2}\right)^{2}-K_{2} \epsilon_{x}\left(3 q_{1}+q_{2}\right)^{2}-2 K_{3} \epsilon_{x}\left(3 q_{1}+q_{2}\right)^{2}>0, \\
1-K_{1} q_{4}^{2}-K_{3} \epsilon_{x}>0, \\
1-K_{2} \epsilon_{x}>0 .
\end{array}
$$

Therefore, if the restrictions of (77) are fulfilled, $\dot{V}$ is strictly negative, and the state vector $q$ exponentially converges to zero after $t>T_{4}$.

The following proposition summarizes the previous discussion, which is the main result of our study.

Proposition 1. Consider the PVTOL aircraft system with an inverted pendular load as described in (9) and in a closed loop with controllers (12), (17a) and (17b), (23), (25), and (49). Then, the closed-loop system is exponentially asymptotically stable provided that the control parameters $c_{1}, c_{2}, c_{3}$, and $c_{4}$ satisfy inequalities (69), and $k_{\alpha_{i}}$ and $k_{y_{i}}$ are selected such that the characteristic polynomial is Hurwitz.
Finally, in Figure 3, the steps' sequence is shown, obtained from the control laws $u_{1}$ and $u_{2}$.

Having designed the control scheme for the PVTOL aircraft system with an inverted pendular load and carried out its convergence analysis, we test its effectiveness through numerical simulations in the following section. It is worth mentioning that it would be ideal for testing the scheme by conducting actual experiments-unfortunately, the construction of the needed prototype is still in progress.

\section{Numerical Simulations}

To test the controllers' performance, we carried out some numerical simulations using the MATLAB-Simulink program, and the results were obtained based on the numerical method of Runge-Kutta of the fourth order with a fixed step of $0.01 \mathrm{~s}$. The physical parameters of the system are $m_{p}=0.2 \mathrm{~kg}, m_{v}=0.8 \mathrm{~kg}, l_{p}=0.2 \mathrm{~m}$, and $g=9.8 \mathrm{~m} / \mathrm{s}^{2}$. Also, the tuning parameters proposed for each controller are listed in Table 1 . Notice that the controller parameter values $u_{2}$ and $v_{2}$ were selected such that the error dynamics is equal to the desired closed-loop polynomial $\left(s^{2}+2 \zeta \omega_{n} s+\omega_{n}^{2}\right)(s+\beta)$. 


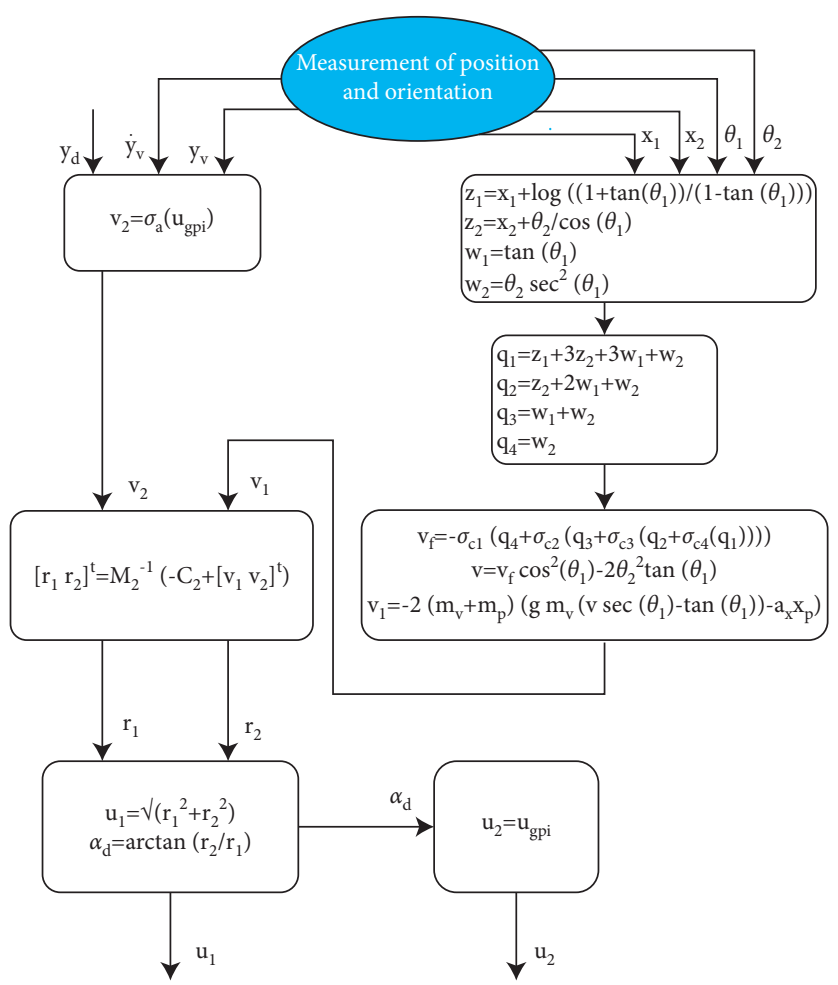

Figure 3: Control strategy sequence.

TABLE 1: Tuning parameters for controllers.

\begin{tabular}{ccc}
\hline Controller & Control parameter & Gain \\
\hline & $L$ & 3 \\
& $k_{\alpha_{0}}$ & 167 \\
& $k_{\alpha_{1}}$ & 299 \\
& $k_{\alpha_{2}}$ & 183 \\
$u_{2}$ & $k_{\alpha_{3}}$ & 62 \\
& $k_{\alpha_{4}}$ & 11 \\
& $a$ & 1.45 \\
& $k_{y_{0}}$ & 167 \\
& $k_{y_{1}}$ & 299 \\
& $k_{y_{2}}$ & 183 \\
$v_{2}$ & $k_{y_{3}}$ & 62 \\
& $k_{y_{4}}$ & 1 \\
& $c_{1}$ & 0.11 \\
$v_{f}$ & $c_{2}$ & 0.24 \\
& $c_{3}$ & 0.49 \\
& $c_{4}$ & 0.99 \\
\hline
\end{tabular}

Besides, the parameters of $v_{f}$ were chosen such that $c_{i}<1 / 2 c_{i+1}$ [40]. Finally, the initial conditions were set as follows: $x_{v}(0)=0.2 \mathrm{~m}, y_{v}(0)=0 \mathrm{~m}, \alpha=0 \mathrm{rad}, \theta=0.1 \mathrm{rad}$, $\dot{x}_{v}=0.15 \mathrm{~m} / \mathrm{s}, \dot{y}_{v}=0 \mathrm{~m} / \mathrm{s}, \dot{\alpha}=0 \mathrm{rad} / \mathrm{s}$, and $\dot{\theta}=0.05 \mathrm{rad} / \mathrm{s}$.

In the first experiment, the control strategy simultaneously carries out height position by performing trajectory regulation, stabilizing the horizontal position and roll angle for the PVTOL and the pendulum angle. In this case, the desired trajectory, $y_{d}$, was proposed as

$$
y_{d}=\frac{1}{\left(1+e^{20-2 t}\right)\left(1+e^{-60+2 t}\right)} .
$$

To test the effectiveness of the control strategy introduced in this study, we carried out a comparison test against the classical PD controller, with gains tuned as $k_{p}=1$ and $k_{d}=4$. Figure 4 shows the outcome of this experiment, where we can observe that both strategies successfully achieve the height position regulation through the trajectory tracking task and the stabilization of the horizontal position, the roll angle, and the pendulum angle. Also, the plot in this figure shows that our controller converges to the desired values faster than the PD controller does. Figure 5 shows the behaviours of the angular velocities, and Figure 6 shows the tracking error between $\alpha$ and $\alpha_{d}$ and the control inputs $u_{1}$ and $u_{2}$.

Using the same setup as before, we run a second experiment, but in this case, the system is affected by external disturbances with parameters fixed as $a_{x}=1.4, a_{y}=0.8$, and $a_{\theta}=2$. Figure 7 shows the corresponding plots, where we can see that our controller is capable of accomplishing height tracking and position regulation and, simultaneously, stabilizing the horizontal position, roll angle, and pendulum angle. Also, it can be seen that the PD controller used in the first experiment regulates the system slower than our controller does and exhibits undesirable oscillations. Therefore, our controller has better performance, maneuverability, and whole stabilization, even when the system is affected by external disturbances. Figure 8 shows the system angular velocities, and Figure 9 shows the tracking error behaviour between $\alpha$ and $\alpha_{d}$ and the control inputs $u_{1}$ and $u_{2}$. Please notice that the proposed control has adequate energy management, according to the obtained performance index $\int u_{i}^{2}$ shown in Figure 10 . 


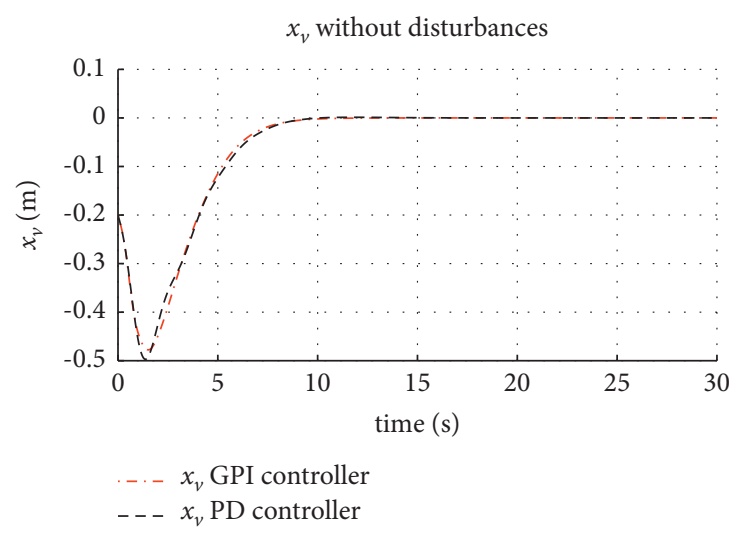

(a)

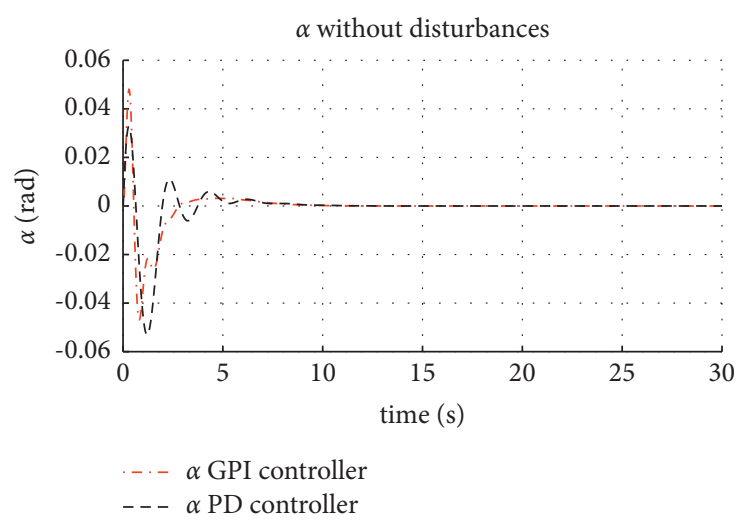

(c)

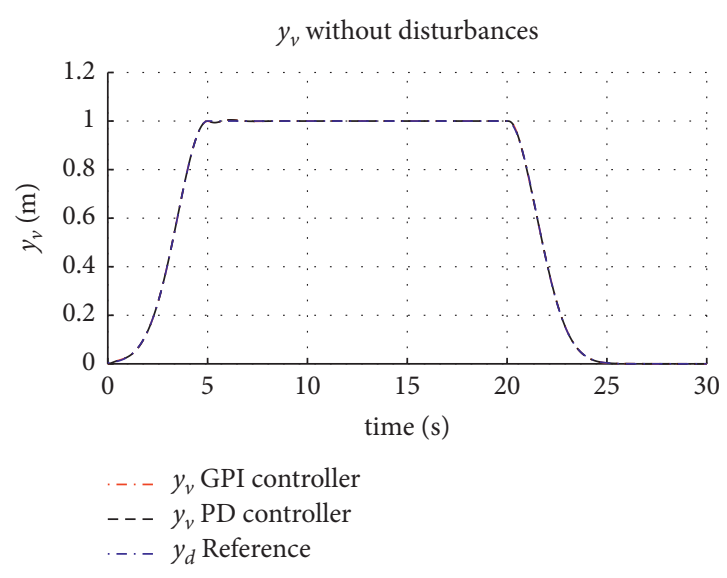

(b)

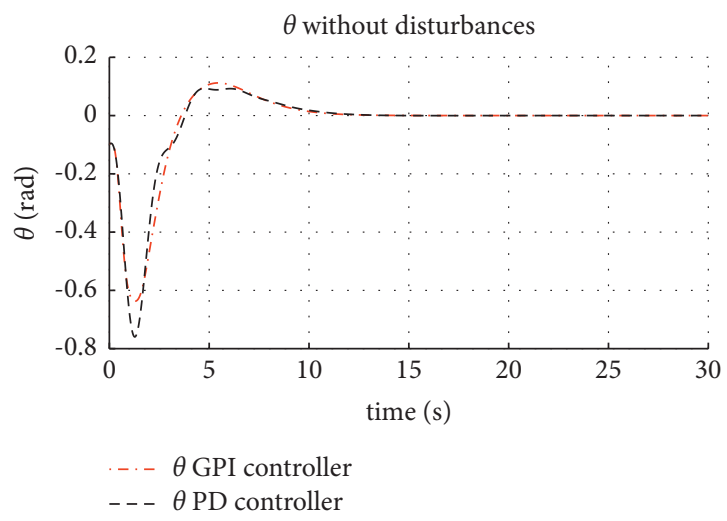

(d)

Figure 4: Comparison between the position closed-loop responses of the proposed controller and PD controller. (a) Behaviour of state $x_{v}$. (b) Behaviour of state $y_{v}$. (c) Behaviour of state $\alpha$. (d) Behaviour of state $\theta$.

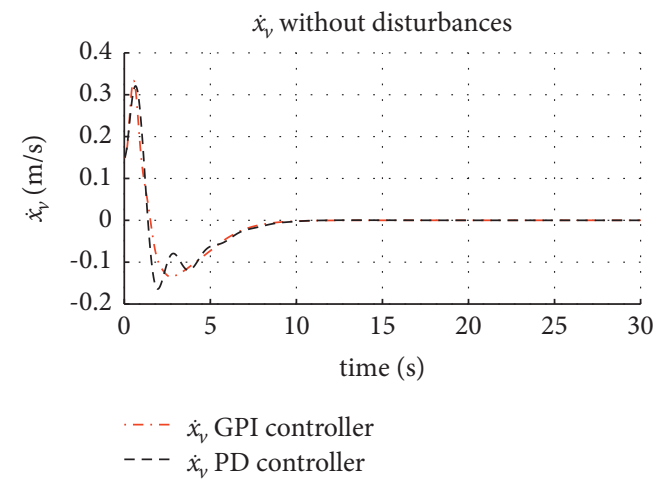

(a)

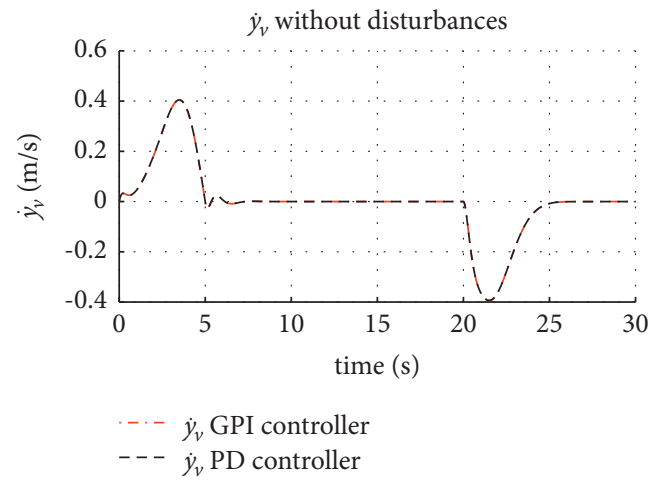

(b)

Figure 5: Continued. 


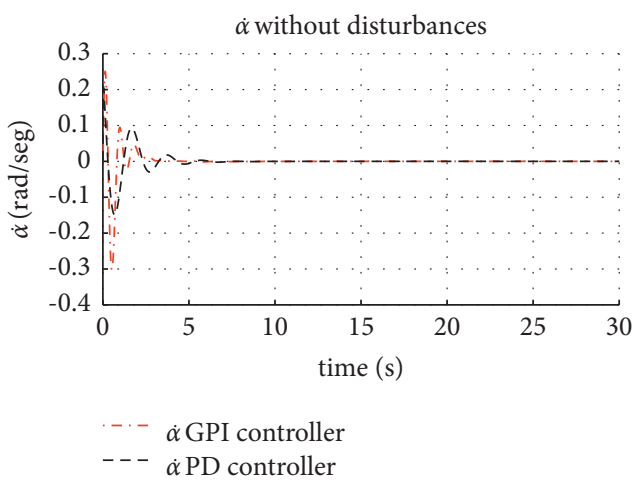

(c)

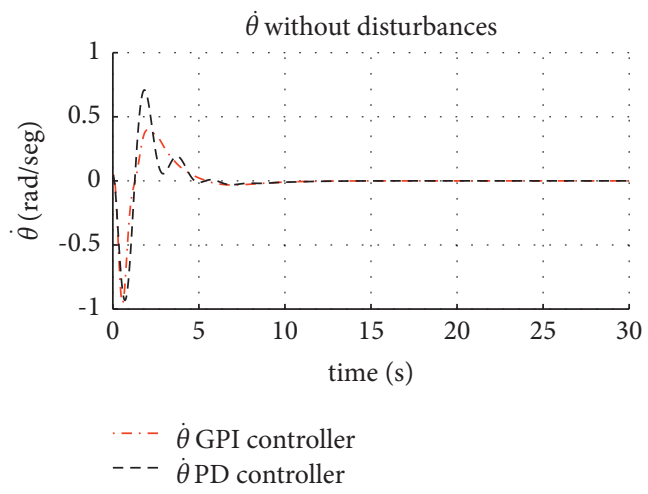

(d)

Figure 5: Angular velocities' closed-loop response of the proposed controller. (a) Behaviour of state $\dot{x}_{v}$. (b) Behaviour of state $\dot{y}_{v}$. (c) Behaviour of state $\dot{\alpha}$. (d) Behaviour of state $\dot{\theta}$.

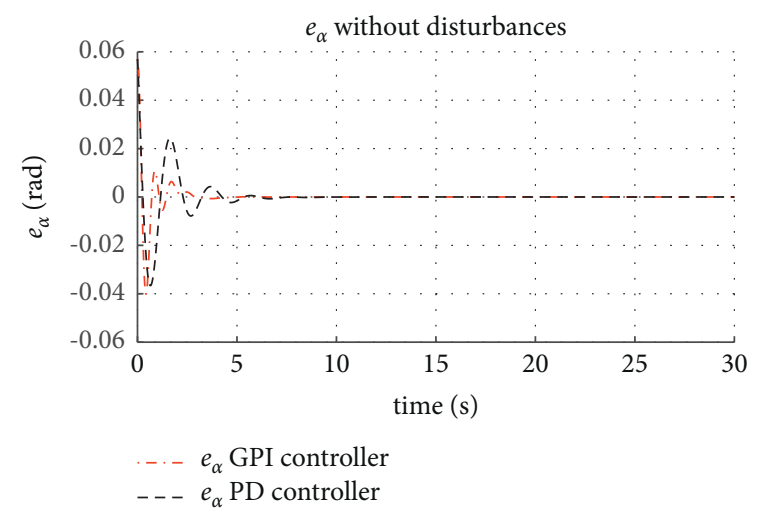

(a)

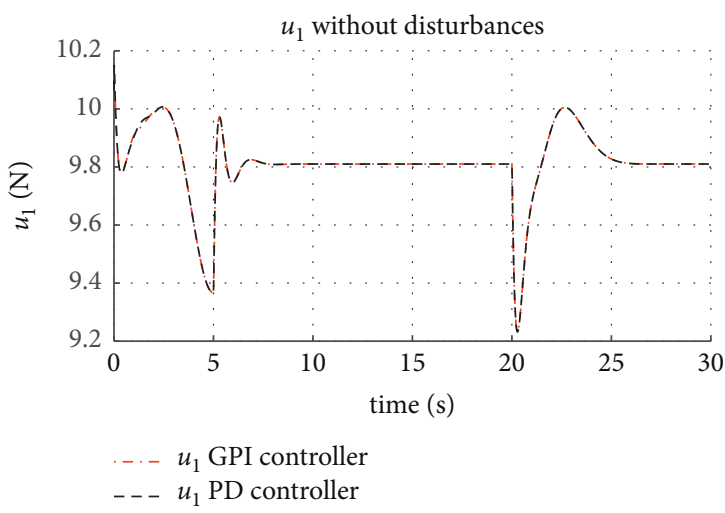

(b)

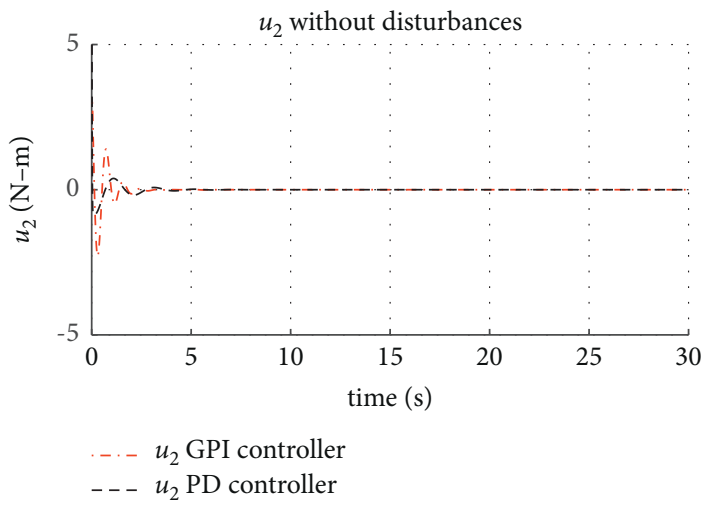

(c)

FiguRE 6: Tracking error of the state $\alpha$ and behavior of controllers $u_{1}$ and $u_{2}$. (a) Error tracking dynamics for $\alpha$. (b) Control action $u_{1}$. (c) Control action $u_{2}$. 


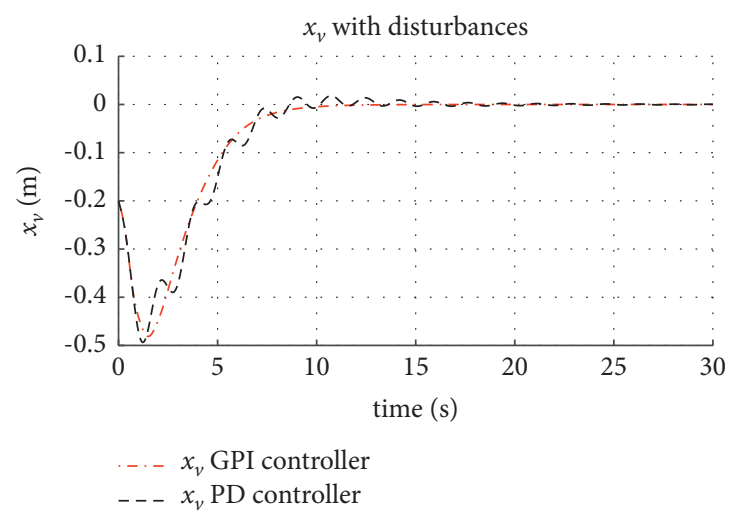

(a)

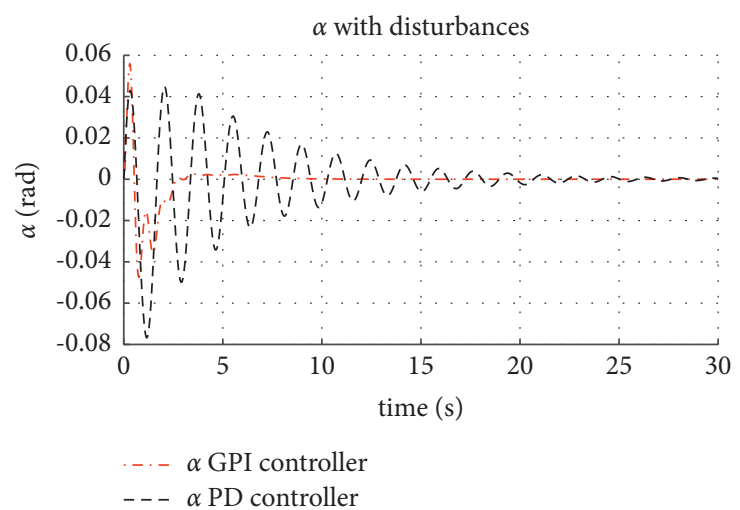

(c)

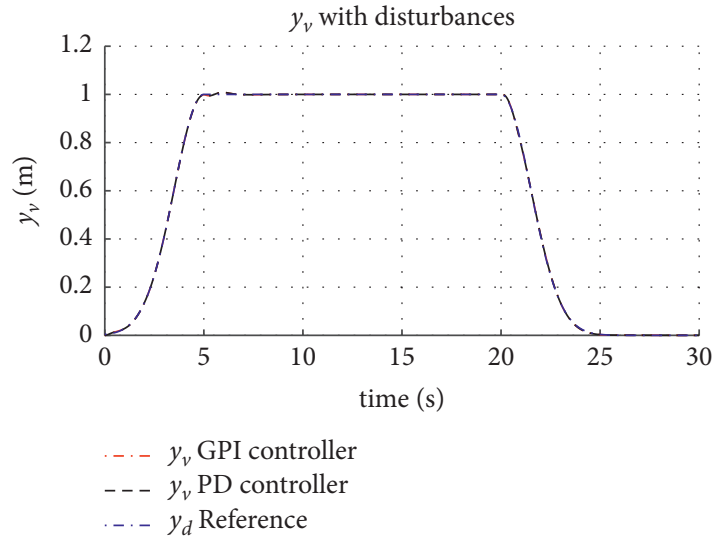

(b)

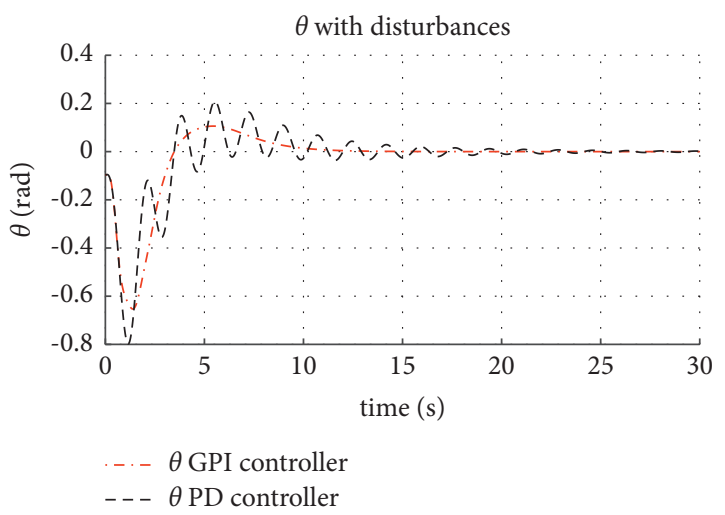

(d)

Figure 7: Position closed-loop response of the proposed controller in the presence of external disturbances. (a) Behaviour of state $\dot{x}_{v}$. (b) Behaviour of state $\dot{y}_{v}$. (c) Behaviour of state $\dot{\alpha}$. (d) Behaviour of state $\dot{\theta}$.

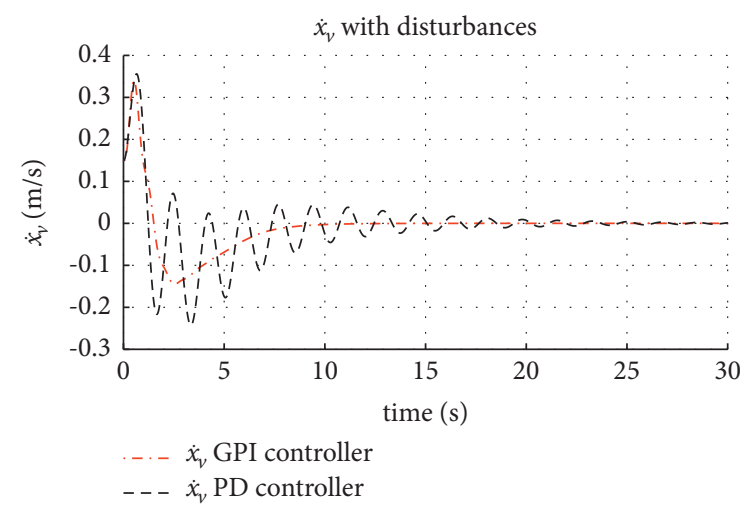

(a)

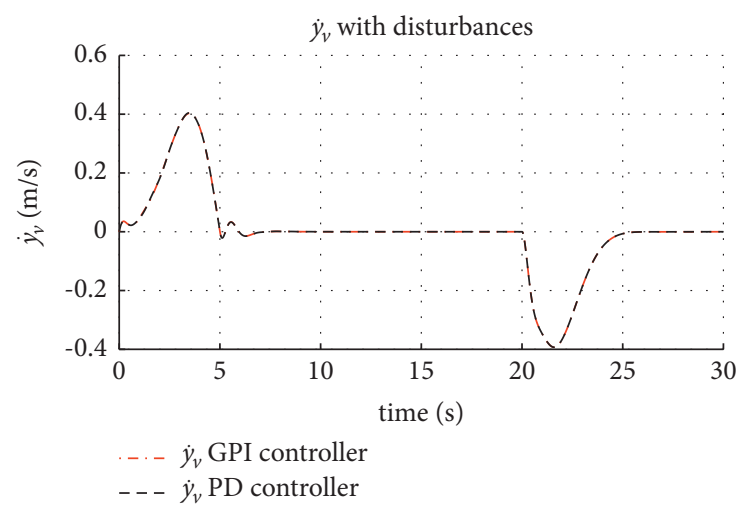

(b)

FIgUre 8: Continued. 


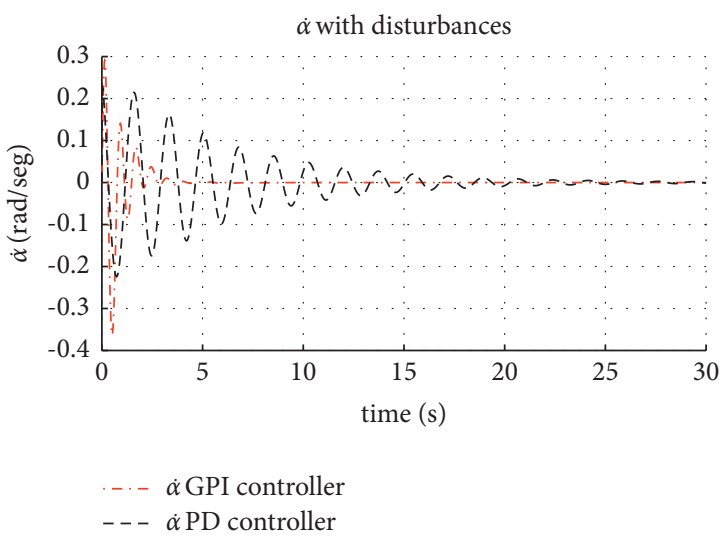

(c)

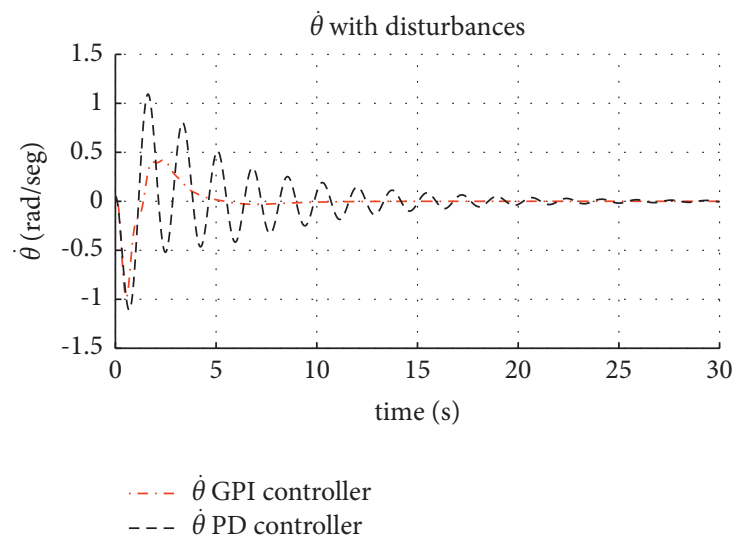

(d)

Figure 8: Angular velocities' closed-loop responses of the proposed controller in the presence of external disturbances. (a) Behaviour of state $\dot{x}_{v}$. (b) Behaviour of state $\dot{y}_{v}$. (c) Behaviour of state $\dot{\alpha}$. (d) Behaviour of state $\dot{\theta}$.

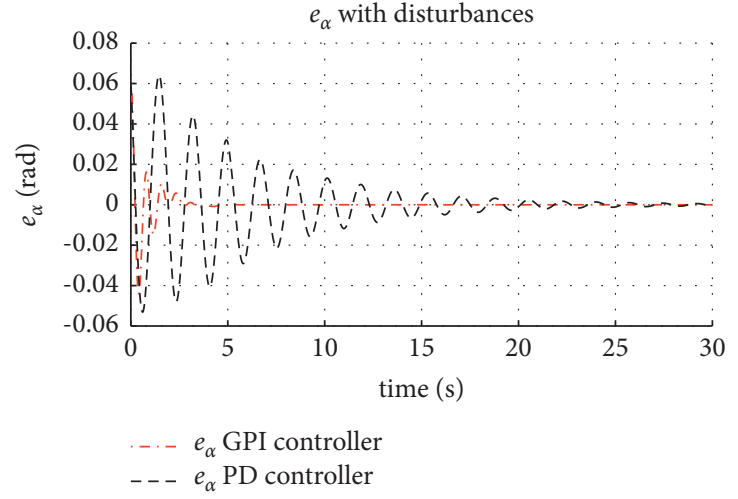

(a)

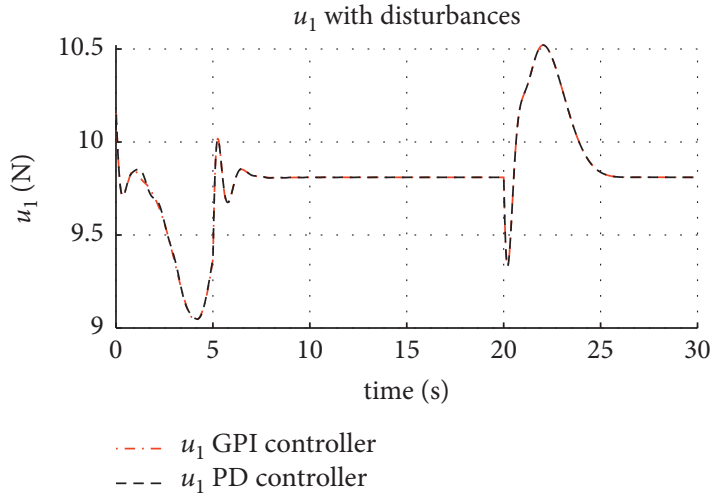

(b)

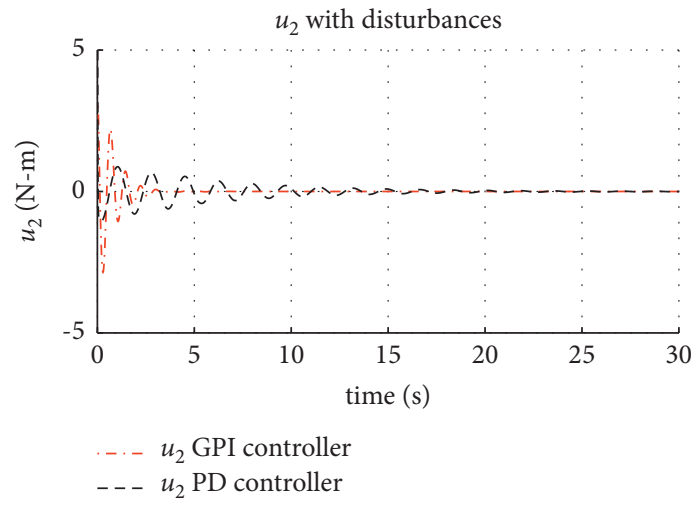

(c)

FIGURE 9: The behaviour of the error tracking of the state $\alpha$ and controllers $u_{1}$ and $u_{2}$, respectively, owing to external disturbances. (a) Error tracking dynamics for $\alpha$. (b) Control action $u_{1}$. (c) Control action $u_{2}$. 


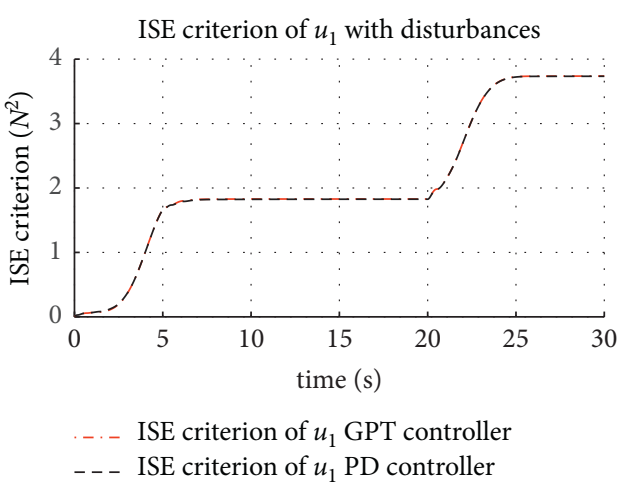

(a)

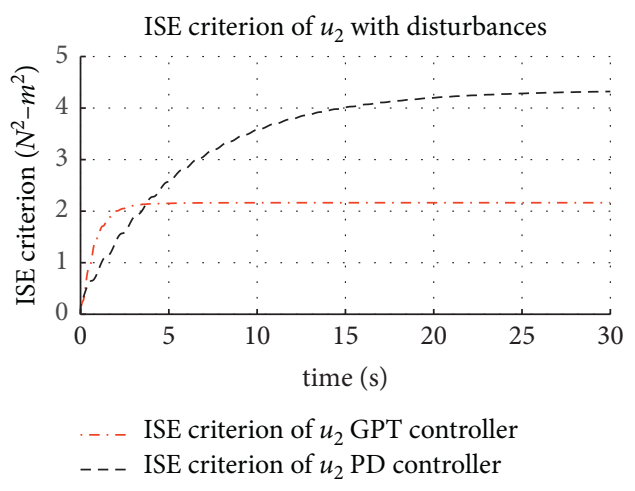

(b)

FIgURE 10: The performance index of the controllers $u_{1}$ and $u_{2}$, respectively, owing to external disturbances. (a) Performance index of the controller $u_{1}$. (b) Performance index of the controller $u_{2}$.

\section{Conclusions}

This work presents a nested saturation function-based controller, combined with a GPI controller, to stabilize the PVTOL aircraft system with an inverted pendular load. The model of this system was derived using Euler-Lagrange formalism. The main contribution consists of using a fictitious control, and then a GPI controller is proposed for the aircraft angle $(\alpha)$. Several linear transformations and coordinate changes were introduced to express the original system to a minimal representation. To accomplish the takeoff and landing maneuvers, we propose a GPI controller to track the desired trajectory. After stabilizing the PVTOL height, the system was represented as a chain of integrators plus nonlinear disturbance, allowing us to use nested saturation functions to design a controller to stabilize the horizontal position and pendulum angle. The stability analysis was carried out using the second method of Lyapunov, using a simple candidate function. Designing the control scheme was not an easy task because the PVTOL system with an inverted pendular load is underactuated, and ensuring the pendulum's upright position makes this problem even harder to solve. We ran numerical simulations to assess the performance of our control scheme, having obtained convincing results. These simulations included a comparison against the well-established PD control strategy. The performance index of both controllers was computed to compare them, and the outcome revealed that our strategy has a better performance than the PD controller. It is important to note that the performance indexes were estimated in the presence of nonlinear perturbations, which means that the proposed controller behaves well even in this undesirable yet unavoidable realistic scenario. It is important to note that the controller, based on a GPI controller and nested saturation functions, allows us to perform takeoff maneuvers in the presence of exogenous disturbances.

In future work, we will explore a design to estimate the disturbance due to wind or robust techniques for parametric uncertainties of the system. In addition, it is worth mentioning that an experimental platform that allows configuring the PVTOL with an inverted pendular load system has been designed, whose construction is in process, Thus, experimental implementation of the control scheme proposed herein is considered.

\section{Data Availability}

No data were used to support this study.

\section{Conflicts of Interest}

The authors declare that there are no conflicts of interest regarding the publication of this article.

\section{Acknowledgments}

This research was funded by Secretaria de Investigación y Posgrado-Instituto Politécnico Nacional grants nos. 20210268, 20210253, and 20211168. C. Alejandro Villaseñor Rios thanks the support from the CONACYT. Cesar Alejandro Villaseñor Rios, Octavio Gutiérrez-Frías, Carlos Aguilar-Ibanez, and Miguel S. Suarez-Castanon are at Instituto Politécnico Nacional and they are the ones to express their gratitude for the support received.

\section{References}

[1] D. J. Block and M. W. Spong, "Mechanical design and control of the pendubot," SAE Transactions, pp. 36-43, 1995.

[2] I. Fantoni and R. Lozano, "Global stabilization of the cartpendulum system using saturation functions," 42nd IEEE International Conference on Decision and Control, vol. 5, pp. 4393-4398, 2003.

[3] C. A. Ibañez and O. G. Frias, "Controlling the inverted pendulum by means of a nested saturation function," Nonlinear Dynamics, vol. 53, no. 4, pp. 273-280, 2008.

[4] C. Aguilar-Ibañez, M. S. Suárez-Castañón, and O. O. Gutiérres-Frias, "The direct lyapunov method for the stabilisation of the furuta pendulum," International Journal of Control, vol. 83, no. 11, pp. 2285-2293, 2010.

[5] M. Ramirez-Neria, Z. Gao, H. Sira-Ramirez, R. GarridoMoctezuma, and A. Luviano-Juarez, "Trajectory tracking for an inverted pendulum on a cart: an active disturbance rejection control approach," in Proceedings of the 2018 Annual American Control Conference (Acc), pp. 4881-4886, Milwaukee, Wisconsin, June 2018. 
[6] M. Nakamura, K. Takaya, H. Ohta, K. Shibayama, and V. Kroumov, "Quadrotor modeling and simulation for industrial application," in Proceedings of the 2019 23rd International Conference on System Theory, Control and Computing (ICSTCC), pp. 37-42, Sinaia, Romania, October 2019.

[7] S. Saeedi, C. Thibault, M. Trentini, and H. Li, "3d mapping for autonomous quadrotor aircraft," Unmanned Systems, vol. 5, no. 3, pp. 181-196, 2017.

[8] A. Salih, M. Moghavvemi, H. Mohamed, and K. Gaeid, "Flight pid controller design for a uav quadrotor," Scientific Research and Essays, vol. 5, no. 23, pp. 3660-3667, 2010.

[9] J. Hauser, S. Sastry, and G. Meyer, "Nonlinear control design for slightly non-minimum phase systems: application to v/stol aircraft," Automatica, vol. 28, no. 4, pp. 665-679, 1992.

[10] C. Aguilar-Ibanez, M. S. Suarez-Castanon, J. Meda-Campaña, O. Gutierrez-Frias, C. Merlo-Zapata, and J. A. MartinezCastro, "A simple approach to regulate a pvtol system using matching conditions," Journal of Intelligent \& Robotic Systems, vol. 98, pp. 1-14, 2019.

[11] I. Fantoni, A. Zavala, and R. Lozano, "Global stabilization of a pvtol aircraft with bounded thrust," Proceedings of the 41st ieee conference on decision and control, vol. 4, pp. 4462-4467, 2002.

[12] A. Zavala-Río, I. Fantoni, and R. Lozano, "Global stabilization of a pvtol aircraft model with bounded inputs," International Journal of Control, vol. 76, no. 18, pp. 1833-1844, 2003.

[13] L. Zou, H. Li, W. Zhao, and L. Zhu, "Imaged-based visual servo control for a Vtol aircraft," Mathematical Problems in Engineering, vol. 2017, Article ID 4806769, 6 pages, 2017.

[14] R. Czyba, M. Lemanowicz, Z. Gorol, and T. Kudala, "Construction prototyping, flight dynamics modeling, and aerodynamic analysis of hybrid vtol unmanned aircraft," Journal of Advanced Transportation, vol. 2018, Article ID 7040531, 17 pages, 2018.

[15] L. Wang and J. Cheng, "Finite-time terminal sliding mode tracking control of a vtol uav via the generalized ndob," Mathematical Problems in Engineering, vol. 2020, Article ID 4651475, 13 pages, 2020.

[16] M. Hehn and R. D'Andrea, "A flying inverted pendulum," in Proceedings of the 2011 IEEE International Conference on Robotics and Automation, pp. 763-770, Shanghai, China, May 2011.

[17] R. Figueroa, A. Faust, P. Cruz, L. Tapia, and R. Fierro, "Reinforcement learning for balancing a flying inverted pendulum," in Proceedings of the 11th World Congress on Intelligent Control and Automation, pp. 1787-1793, Shenyang, China, June 2014.

[18] D. S. Maughan, I. Erekson, and R. Sharma, "Using extended kalman filter for robust control of a flying inverted pendulum," in Proceedings of the 2015 IEEE Signal Processing and Signal Processing Education Workshop (sp/spe), pp. 101-106, Snowbird, UT, USA, August 2015.

[19] D. S. Maughan, I. T. Erekson, and R. Sharma, "Flying inverted pendulum trajectory control on robust intelligent sensing and control multi-agent analysis platform," in Proceedings of the 2015 International Conference on Unmanned Aircraft Systems (ICUAS), pp. 1279-1284, Denever, CO, USA, June 2015.

[20] C. Zhang, H. Hu, D. Gu, and J. Wang, "Cascaded control for balancing an inverted pendulum on a flying quadrotor," Robotica, vol. 35, no. 6, pp. 1263-1279, 2017.

[21] M. M. Nicotra, E. Garone, R. Naldi, and L. Marconi, "Nested saturation control of an uav carrying a suspended load," in Proceedings of the 2014 American Control Conference, pp. 3585-3590, Portland, OG, USA, June 2014.
[22] I. H. Pizetta, A. Brandão, and M. Sarcinelli-Filho, "Modelling and control of a pvtol quadrotor carrying a suspended load," in Proceedings of the 2015 International Conference on Unmanned Aircraft Systems (ICUAS), pp. 444-450, Denever, CO, USA, June 2015.

[23] C. Merlo-Zapata, C. Aguilar-Ibanez, O. Gutiérrez-Frías, M. Antonio-Cruz, C. Márquez-Sánchez, and M. SuarezCastanon, "A robust control scheme for a pvtol system subject to wind disturbances," Complexity, vol. 2020, Article ID 3510396, 11 pages, 2020.

[24] A. Moeini, A. F. Lynch, and Q. Zhao, "A backstepping disturbance observer control for multirotor uavs: theory and experiment," International Journal of Control, vol. 21, pp. 1-15, 2021.

[25] B. Chen, C. Lin, X. Liu, and K. Liu, “Adaptive fuzzy tracking control for a class of mimo nonlinear systems in nonstrictfeedback form," IEEE Transactions on Cybernetics, vol. 45, no. 12, pp. 2744-2755, 2014.

[26] M. A. Jaradat, M. Al-Fandi, O. Alkhatib, and Y. Sardahi, "Intelligent control of miniature holonomic vertical take-off and landing robot," Journal of Intelligent \& Fuzzy Systems, vol. 29, no. 3, pp. 1209-1217, 2015.

[27] S. C. Tong, "Adaptive fuzzy control for uncertain nonlinear systems," Journal of Control and Decision, vol. 6, no. 1, pp. 30-40, 2019.

[28] C. Aguilar-Ibanez, H. Sira-Ramirez, M. Suarez-Castanon, and R. Garrido, "Robust trajectory-tracking control of a pvtol under crosswind," Asian Journal of Control, vol. 21, no. 3, pp. 1293-1306, 2019.

[29] Q. Yao, "Robust constrained trajectory tracking control for a pvtol aircraft subject to external disturbances," International Journal of Systems Science, vol. 52, pp. 1-13, 2021.

[30] S. Bouabdallah, "Design and control of quadrotors with application to autonomous flying," Technical Report, Epfl, Lausanne, Switzerland, 2007.

[31] Y. Lozano Hernández, O. Gutiérrez Frías, N. Lozada-Castillo, and A. Luviano Juárez, "Control algorithm for taking off and landing manoeuvres of quadrotors in open navigation environments," International Journal of Control, Automation and Systems, vol. 17, no. 9, pp. 2331-2342, 2019.

[32] M. Fliess, R. Marquez, and E. Delaleau, "State feedbacks without asymptotic observers and generalized pid regulators," in Nonlinear Control in the Year 2000, pp. 367-384, Springer, Berlin, Germany, 2001.

[33] B. R. Angara and M. Tripathi, "Design of gpi controller for current feedback multilevel cascaded inverter," in Proceedings of the 2016 Biennial International Conference on Power and Energy Systems: Towards Sustainable Energy (PESTSE), pp. 1-5, Bangalore, India, January 2016.

[34] H. Sira-Ramírez, A. Luviano-Juárez, M. Ramírez-Neria, and E. W. Zurita-Bustamante, Active Disturbance Rejection Control of Dynamic Systems: A Flatness Based Approach, Butterworth-Heinemann, Oxford, UK, 2018.

[35] C. Aguilar Ibanez, O. Gutiérrez Frías, and M. S. Suarez Castanon, "Controlling the strongly damping inertia wheel pendulum via nested saturation functions," Computación $Y$ Sistemas, vol. 12, no. 4, pp. 436-449, 2009.

[36] C. Aguilar Ibanez, J. C. Martinez Garcia, A. Soria Lopez, J. d. J. Rubio, and M. S. Suarez Castanon, "Stabilization of the inverted cart-pendulum system with linear friction," IEEE Latin America Transactions, vol. 16, no. 6, pp. 1650-1657, 2018.

[37] P. Castillo, A. Dzul, and R. Lozano, "Real-time stabilization and tracking of a four-rotor mini rotorcraft," IEEE 
Transactions on Control Systems Technology, vol. 12, no. 4, pp. 510-516, 2004.

[38] C. Aguilar-Ibañez, J. C. Martínez-García, A. Soria-López, and J. Rubio, "On the stabilization of the inverted-cart pendulum using the saturation function approach," Mathematical Problems in Engineering, vol. 2011, Article ID 856015, 14 pages, 2011.

[39] R. Olfati-Saber, "Fixed point controllers and stabilization of the cart-pole system and the rotating pendulum," in Proceedings of the 38th IEEE Conference on Decision and Control, pp. 1174-1181, Phenoix, AZ, USA, December 1999.

[40] A. R. Teel, "Global stabilization and restricted tracking for multiple integrators with bounded controls," Systems \& Control Letters, vol. 18, no. 3, pp. 165-171, 1992. 